\title{
Pharmacophore-Guided Identification of Natural Products as Potential Inhibitors of Mycobacterium ulcerans Cystathionine $\gamma$-synthase MetB
}

\author{
Samuel K. Kwofie 1,2,3,*®D, Nigel N. O. Dolling 1,4过, Emmanuel Donkoh ${ }^{1,4}$, Godwin M. Laryea ${ }^{1}$, Lydia Mosi ${ }^{2}$, \\ Whelton A. Miller III ${ }^{3,5}$, Michael B. Adinortey ${ }^{6}\left(\mathbb{D}\right.$ and Michael D. Wilson ${ }^{3,4}$ \\ 1 Department of Biomedical Engineering, School of Engineering Sciences, College of Basic and Applied \\ Sciences, University of Ghana, Legon, Accra LG 77, Ghana; ndolling5@gmail.com (N.N.O.D.); \\ emmanueldonkoh11@gmail.com (E.D.); gomlaryea@gmail.com (G.M.L.) \\ 2 West African Center for Cell Biology and Infectious Pathogens, Department of Biochemistry, Cell and \\ Molecular Biology, University of Ghana, Legon, Accra LG 54, Ghana; LMosi@ug.edu.gh \\ 3 Department of Medicine, Loyola University Medical Center, Maywood, IL 60153, USA; \\ wheltonm@seas.upenn.edu (W.A.M.III); MWilson@noguchi.ug.edu.gh (M.D.W.) \\ 4 Department of Parasitology, Noguchi Memorial Institute for Medical Research (NMIMR), College of Health \\ Sciences (CHS), University of Ghana, Legon, Accra LG 581, Ghana \\ 5 Department of Chemical and Biomolecular Engineering, School of Engineering and Applied Science, \\ University of Pennsylvania, Philadelphia, PA 19104, USA \\ Citation: Kwofie, S.K.; \\ 6 Department of Biochemistry, School of Biological Sciences, University of Cape Coast, \\ Cape Coast CC 033, Ghana; madinortey@ucc.edu.gh \\ * Correspondence: skkwofie@ug.edu.gh
}

Dolling, N.N.O.; Donkoh, E.; Laryea, G.M.; Mosi, L.;

Miller, W.A., III; Adinortey, M.B.;

Wilson, M.D. Pharmacophore-Guided

Identification of Natural Products as

Potential Inhibitors of Mycobacterium ulcerans Cystathionine $\gamma$-synthase

MetB. Computation 2021, 9, 32.

https://doi.org/10.3390/

computation 9030032

Academic Editor: Shizuka Uchida

Received: 21 January 2021

Accepted: 4 March 2021

Published: 12 March 2021

Publisher's Note: MDPI stays neutral with regard to jurisdictional claims in published maps and institutional affiliations.

Copyright: (c) 2021 by the authors. Licensee MDPI, Basel, Switzerland. This article is an open access article distributed under the terms and conditions of the Creative Commons Attribution (CC BY) license (https:// creativecommons.org/licenses/by/ $4.0 /)$.

\begin{abstract}
Buruli ulcer caused by Mycobacterium ulcerans (M. ulcerans) is identified by a pain-free cyst or edema which develops into a massive skin ulcer if left untreated. There are reports of chemoresistance, toxicity, noncompliance, and poor efficacy of current therapeutic options. Previously, we used cheminformatics approaches to identify potential antimycobacterial compounds targeting major receptors in $M$. ulcerans. In this paper, we sought to identify potential bioactive compounds by targeting Cystathionine gamma-synthase (CGS) MetB, a key receptor involved in methionine synthesis. Inhibition of methionine synthesis restricts the growth of M. ulcerans. Two potent inhibitors Juglone ( $\left.\mathrm{IC}_{50} 0.7+/-0.7 \mu \mathrm{mol} / \mathrm{L}\right)$ and 9-hydroxy-alpha-lapachone $\left(\mathrm{IC}_{50} 0.9+/-0.1 \mu \mathrm{mol} / \mathrm{L}\right)$ were used to generate 3D chemical feature pharmacophore model via LigandScout with a score of 0.9719 . The validated model was screened against a pre-filtered library of 2530 African natural products. Compounds with fit scores above 66.40 were docked against the structure of CGS to generate hits. Three compounds, namely Gentisic 5-O glucoside (an isolate of African tree Alchornea cordifolia), Isoscutellarein (an isolate of Theobroma plant) and ZINC05854400, were identified as potential bioactive molecules with high binding affinities of $-7.1,-8.4$ and $-8.4 \mathrm{kcal} / \mathrm{mol}$ against CGS, respectively. Novel structural insight into the binding mechanisms was elucidated using LigPlot+ and molecular dynamics simulations. All three molecules were predicted to possess antibacterial, anti-ulcerative, and dermatological properties. These compounds have the propensity to disrupt the methionine synthesis mechanisms with the potential of stagnating the growth of M. ulcerans. As a result of reasonably good pharmacological profiling, the three drug-like compounds are potential novel scaffolds that can be optimized into antimycobacterial molecules.
\end{abstract}

Keywords: Buruli ulcer; Cystathionine $\gamma$-synthase MetB; Mycobacterium ulcerans; natural products; molecular docking; pharmacophore modeling; antimycobacterial

\section{Introduction}

Mycobacterium ulcerans belongs to the slow-growing environmental mycobacteria family which secretes mycolactone, a toxin that has a strong cytotoxic activity. Buruli ulcer 
(BU) is an infectious, flesh-eating ailment that affects the skin and subcutaneous tissues [1]. Mycolactone, when present in the human system, induces cell death, necrosis of several cell types, and suppresses the immune response. The disease is characterized by a pain-free cyst, plaque, or edema which develops into a massive skin ulcer if left untreated [2]. BU currently ranks third among mycobacterial diseases that affect humans, with leprosy and tuberculosis placing first and second, respectively. Yet among the three, BU is the least understood [1,2].

$\mathrm{BU}$ is prevalent in rural areas of Asia, tropical countries in Africa, Australia, and the Americas with a high focal distribution along water bodies. More than 20,000 cases were reported over the last decade in West Africa, which records the highest prevalence rates; Côte d'Ivoire, Benin, Nigeria, and Ghana are leading the charts $[1,3,4]$. The transmission mode of M. ulcerans is reportedly less understood but the disease is considered to be related to activities around water bodies $[1,2,5]$. It is speculated that environmental $M$. ulcerans enters the body through small cuts in the skin from direct contact with contaminated soil, water, or vegetation, and in some cases, is transmitted through biting water-borne insects [6].

M. ulcerans is an acid-fast bacillus that has a genome size of 5,805,761 base pairs, 4160 protein-coding genes, 771 pseudogenes and consists of two circular replicons. Cystathionine gamma-synthase MetB (CGS), a protein encoded by a gene of $M$. ulcerans, exists as a homotetramer comprising four identical monomers (Chains A, B, C and D) with each having its active site. Present in each active site is the cofactor pyridoxal phosphate (PLP), responsible for activating the protein. More significantly, CGS plays a vital role in the synthesis of methionine, an integral requirement for the growth of M. ulcerans, where it is involved in the early committed step in the methionine biosynthesis pathway. It works as a transferase catalyzing the irreversible reaction between $\mathrm{O}$-succinyl-homoserine and cysteine to produce cystathionine and succinate. It is also involved in selenoamino acid and sulfur metabolism [7]. Therefore, CGS MetB is considered a crucial drug target because of its important role in methionine synthesis. Inhibition of methionine synthesis could restrict the development of $M$. ulcerans [8].

Current drugs recommended by the World Health Organization (WHO) for the treatment of BU are Rifampicin, Streptomycin, and Clarithromycin [9]. These drugs have a long treatment duration and could lead to side effects such as hearing impairment, kidney problems, skin rashes, and vomiting $[10,11]$. Natural products possess great chemical and structural diversity, biochemical specificity, and other molecular properties that make them promising leads for drug discovery [12]. These unique properties distinguish them from synthetic and combinatorial compound libraries and inspire novel discoveries in chemistry, biology, and medicine [13]. There is a long-term history of usage of natural products and wider public acceptance of drugs of natural product origin [14,15].

Since CGS has been suggested as a drug target [7], the reported work aimed to identify drug-like biomolecules which have the potential to inhibit CGS. Inhibiting the CGS could disrupt the synthesis of methionine critical for the growth of M. ulcerans. This study sought to predict potential novel inhibitors by using ligand-based pharmacophore screening and molecular docking of natural products originating from Africa. In addition, elucidate novel mechanisms of binding and biological activity of the proposed compounds.

\section{Materials and Methods}

\subsection{Target Retrieval and Preparation}

The 3D X-ray crystallographic structures of CGS of M. ulcerans and homolog CGS of Helicobacter pylori were obtained from Research Collaboratory for Structural Bioinformatics (RCSB) Protein Data Bank (PDB) [16]. For the study, chain A of each 3D crystal structural coordinate file was used. Two experimentally elucidated structures of CGS MetB from $M$. ulcerans are available. The structure with PDB ID 3QI6 is bound covalently to PLP (cofactor) and the other with PDB ID 3QHX is bound covalently to both PLP and 4-(2-hydroxyethyl)1-piperazine ethanesulfonic acid (HEPES) [7]. To select which structure to use for this 
study, the resolutions and R-values were considered. Resolution describes the measure of the quality of data found on the crystal containing a protein. It also includes measuring the level of detail seen upon electron density map calculation and the diffraction pattern. As a general rule of thumb, a premium is placed on structures with high resolutions [16]. The R-value denotes the measure of the quality of the atomic model attained from the crystallographic data. The closer a structure's R-value is to 0 the better [17]. PyMOL 1.9.7 $[18,19]$ was used to analyze the 3D structures and to remove all attached ligands as well as water molecules in preparation for further downstream analysis. Missing residues in the solved structural files were incorporated using the Swiss-PdbViewer [20]. The resulting structure was energy minimized in preparation for downstream analysis using grompp embedded in GROMACS [21] with default settings for 5000 steps.

\subsection{Compound Selection for Pharmacophore Generation}

After an exhaustive literature search, we were unable to identify inhibitors specifically for CGS of M. ulcerans. This led to the exploration of the homologs of CGS of M. ulcerans using BLAST from NCBI [22]. CGS of H. pylori was found to be a homolog of CGS of M. ulcerans with a sequence identity of $49.34 \%$. CGS of $H$. pylori is functionally similar to that of $M$. ulcerans since it also catalyzes the creation of L-cystathionine from O-succinyl-L-homoserine (OSHS) and L-cysteine (UniProtKB ID: Q1M0P5; METB_HELPX) [23]. Table 1 shows that CGS of H. pylori has five known inhibitors comprising Juglone [24], $\alpha$-Lapachone, Yangambin, Paulownin, and 9-Hydroxy- $\alpha$-Lapachone [25]. However, to ascertain whether these inhibitors would be suitable for the target protein (CGS of M. ulcerans), the structures of both CGS of $H$. pylori and CGS of M. ulcerans were first superimposed to determine similarities between their binding pockets. The inhibitors were then docked in each protein structure to determine binding mechanisms and consistency within the active site.

Table 1. The five known inhibitors of CGS of Helicobacter pylori with names, $\mathrm{IC}_{50}$ values, and structures.

\begin{tabular}{|c|c|c|}
\hline Compound & $\mathrm{IC}_{50}(\mu \mathrm{M})$ & Structure \\
\hline Juglone & $7 \pm 0.7$ & \\
\hline$\alpha$-Lapachone & $11 \pm 3$ & \\
\hline 9-Hydroxy- $\alpha$-Lapachone & $9 \pm 1$ & \\
\hline Paulownin & $19 \pm 2$ & \\
\hline
\end{tabular}


Table 1. Cont.

\begin{tabular}{|c|c|c|}
\hline Compound & $\mathrm{IC}_{50}(\mu \mathrm{M})$ & Structure \\
\hline Yangambin & $27 \pm 6$ & \\
\hline
\end{tabular}

\subsection{Ligand-Based Pharmacophore Virtual Screening}

LigandScout version 4.3 [26] was used for ligand-based pharmacophore virtual screening. The 2D structures of the inhibitors were retrieved from the ZINC database [27] in Structure Data File (SDF) formats and loaded into LigandScout's Ligand-Based Modeling Perspective v4.3 [26]. The default settings of OMEGA best were used in the generation of ligand conformations with 200 conformations being the maximum limit set per molecule [28].

\subsection{Pre-Filtering of the Library for Pharmacophore-Based Screening}

A unified library comprising 4067 natural products obtained from AfroDb and NANPDB was used for virtual screening. AfroDb and NANPDB were composed of 885 and 3182 compounds, respectively. The library was filtered using Free ADME-Tox Filtering (FAFDrugs4) [29] to eliminate less drug-like compounds based on their physicochemical profiles. The 2530 output compounds from the filtering were employed in pharmacophore-based virtual screening.

\subsection{Pharmacophore-Based Screening of the Library}

A total of 2530 pre-filtered compounds were used for pharmacophore-based virtual screening via LigandScout v.4.3 [26] by screening against the validated pharmacophore model. The compounds were screened after conversion from ".sdf" to ".ldb".

\subsection{Validation of AutoDock Vina}

2.6.1. Superimposition of Co-Crystallized with Re-Docked Complexes

The ligand 4-(2-hydroxyethyl)-1-piperazine ethanesulfonic acid (HEPES) was extracted from the co-crystallized structure of CGS of M. ulcerans and re-docked into the active site. Using LigAlign [30], the predicted binding pose of the HEPES ligand was superimposed with the experimentally determined pose of the co-crystallized structure of the protein.

\subsubsection{ROC Curve Analysis}

The five active compounds of CGS from $H$. pylori together with their decoys were screened against CGS from $M$. ulcerans. The ROC curve and Area Under Curve (AUC) were generated using easyROC [31]. The ROC curve is quantified by the calculation of the Area under the Curve (AUC) with values between the range of 0 and 1 . Another parameter used by LigandScout 4.3 is the Enrichment Factor (EF). EF implies "a ratio of the observed fraction of active compounds in the top few percent of a virtual screen to that expected by random selection" [32]. A good pharmacophore model should be able to significantly distinguish actives from a library composed of inactives and actives. The inactives are decoys of the five known inhibitors. Decoys share similar physical features with the known inhibitors or actives but different chemical structures [33]. 


\subsection{Virtual Screening of the Library}

AutoDock Vina interfaced with PyRx v.0.8 [34] was used for all the virtual screening. The pharmacophore hits obtained from screening the pharmacophore model against the filtered library were virtually screened against the energy minimized protein, CGS of $M$. ulcerans. The pharmacophore hits were imported as ".sdf" format into AutoDock Vina, energy minimized, and converted to ".pdbqt" format. The energy minimization involved the use of the default settings comprising Universal Force Field (UFF) and conjugate gradients for optimization algorithm with a total number of 200 steps. The AutoDock Vina search space center had $X, Y$, and $Z$ coordinates, which were set to the spatial coordinates of $4.8945 \AA,-23.7922 \AA$, and $-37.3622 \AA$, respectively. Grid box dimensions of $25.00 \AA \times 25.00 \AA \times 25.00 \AA$ covered the binding site region. The default exhaustiveness of 8 for AutoDock Vina calculations was used. Ligands that firmly docked in the binding site of the target were selected. Ligands that were not fitted firmly within the binding pocket were eliminated from future analysis.

\subsection{Protein-Ligand Interaction}

LigPlot+ [35] was employed in the characterization of the ligand-protein interactions as $2 \mathrm{D}$ schematic diagrams using default settings.

\subsection{Absorption, Distribution, Metabolism, Excretion, and Toxicity (ADMET) Prediction}

SwissADME [36] and AdmetSAR [37] were used for Absorption, Distribution, Metab olism, Excretion, and Toxicity (ADMET) predictions. Ligands in SMILES format were used to generate the pharmacological profiles. Both SwissADME [36] and AdmetSAR [37] provide access to parameters and predictive models for the computation of pharmacokinetics, physicochemical properties, drug-likeness, and toxicity of small molecules. The parameters that were used included (but not limited to): polarity $\left(20 \AA^{2}<\right.$ TPSA $<130 \AA^{2}$ ); solubility $(0<\log S(E S O L)<6)$; flexibility $(0<$ number of rotatable bonds $<9)$; lipophilicity $(-4.0<$ LOG P $<+5.0)$; size $(150 \mathrm{~g} / \mathrm{mol}<$ molecular weight $<500 \mathrm{~g} / \mathrm{mol})$; hydrogen bond donors $\leq 5$; and hydrogen bond acceptors $\leq 1$ [36]. Lipinski's rule of five is based on a set of rules which describe whether a particular compound is "drug-like" or "orally active" [38]. The rule is based on physicochemical parameter ranges for a compound having a molecular weight $\leq 500$ Daltons; hydrogen bond donors $\leq 5$; a logarithm of $\mathrm{n}$-octanol/ water partition $\leq 5$; and hydrogen bond acceptors $\leq 10$. A compound may be considered less "orally inactive" and "drug-like" if it violates more than two rules.

\subsection{Prediction of Activity Spectra for Substances and Structural Similarity Analogues (PASS)}

Prediction of Activity Spectra for Substances (PASS) [39] was used for the prediction of biological activity based on Bayesian models. Additionally, the hits were screened via the DrugBank [40] to identify similar compounds, derivatives or analogs with antimycobacterial activity.

\subsection{Molecular Dynamics Simulation}

All molecular dynamics (MD) simulations were executed at $100 \mathrm{~ns}$ using GROMACS version 2018 [21] with the SPC water model. The Optimized Potentials for Liquid Simulations (OPLS) / All Atom (AA) force field was used for the simulation of the protein alone, whereas the GROMOS96 43a1 force field was employed in the protein-ligand complexes simulations. All MDs were executed on a supercomputing system. The protein topology files were generated for the GROMACS OPLS/AA force field whereas that of the docked complexes were generated utilizing the PRODRG2 using the settings: 'Yes' for 'Chirality, 'Full' for 'Charges' and 'No' for EM. Simulations were carried out in a $1 \mathrm{~nm}$ dodecahedron box for the application of periodic boundary conditions, solvated and electro-neutralized with the addition of 13 sodium atoms. Thereafter, energies were minimized in 1100 steps. Equilibration followed by standardization of the temperature of the system to the desired 
value for simulation $(300 \mathrm{~K})$ and the application of pressure to the system towards the desired density of $1000 \mathrm{~kg} / \mathrm{m}^{3}$. All graphs were plotted using Xmgrace [41].

\subsection{Molecular Mechanics Poisson-Boltzmann Surface Area Binding Free Energy Calculations}

MM-PBSA was employed in the computation of the binding energies of the ligandprotein complexes utilizing g-mmpbsa over $100 \mathrm{~ns}$ for the MD simulation using GROMACS [42]. Graphs resulting from the MM-PBSA calculations were plotted using the $R$ programming package. The energy terms were calculated over a $100 \mathrm{~ns}$ production run, taking 100 snaps over $1 \mathrm{~ns}$ interval with default settings.

\section{Results and Discussion}

\subsection{Target Description}

Cystathionine gamma synthase exists as a homotetramer possessing two individually wrapped homodimers each bearing two actives sites. Within each active site is the cofactor PLP, which binds tightly to the Lys208 moiety and is stabilized by Ser205, Asp183, Asn158, Met87 and Gly86 through hydrogen bond interactions. There are two structures with PDB IDs 3QI6 and PDB ID 3QHX) [16]. The structure with PDB ID 3QHX was preferred over 3QI6 because of a higher resolution value of $1.65 \AA$ compared to $1.91 \AA$; and R-values of 0.181 and 0.148 as compared to 0.241 and 0.200 , respectively. Additionally, the HEPES ligand was found to be only fully resolved in Chain A of the 3QHX structure. On the other hand, 3QI6 did not contain the HEPES ligand, hence was not selected for further downstream analysis.

\subsection{Ligand-Based Pharmacophore Virtual Screening}

The structural alignment between the 3D protein structures of CGS of H. pylori and M. ulcerans yielded an RMSD of $0.66 \AA$, which was less than the $1.5 \AA$ threshold to be considered as a successful pose (Figure 1) [43]. Consequently, a downstream analysis was undertaken with the inhibitors from CGS of H. pylori.

\subsubsection{Pharmacophore Generation}

For pharmacophore generation, inhibitors with $\mathrm{IC}_{50}<10.0 \mu \mathrm{M}$ were used to generate the pharmacophore features. Thus, Juglone and 9-Hydroxy- $\alpha$-lapachone with $\mathrm{IC}_{50}$ values $0.7+/-0.7$ and $0.9+/-0.1 \mu \mathrm{mol} / \mathrm{L}$, respectively, were used for pharmacophore generation. All the five inhibitors together with their respective decoys were used to validate the pharmacophore model. LigandScout allows the generation of 3D pharmacophores from structural data of ligands rapidly and transparently in a very convenient and automated way [26]. LigandScout implements either the shared- or the merged-feature models for the generation of pharmacophores. The Shared feature model setting selects only features common to training-set molecules. It generates only a few features expected to represent the general binding mechanism of the training set ligands. The merged-feature model setting, on the other hand, selects all features present on each ligand even if those features are not common to most molecules within the training-set. The pharmacophore model was generated with a model score of 0.9719 using a shared feature setting based on the overlap of pharmacophoric features of the two training set compounds of Juglone and 9-hydroxy-lapachone. The features generated via the pharmacophore modeling were 3 hydrogen bond acceptors, 1 hydrogen bond donor, 1 aromatic ring, and 1 hydrophobic interaction (Figure 2).

\subsubsection{Validation of Pharmacophore Model}

The receiver operating characteristic (ROC) curve served to validate the pharmacophore model. Five inhibitors of CGS of $H$. pylori $[23,24]$ were used to generate 250 decoys, with 50 decoys for each inhibitor. DUD-E served as a source for the decoys [33]. A library comprising the decoys and the five active compounds labelled "inactives" and "actives", respectively, was screened against the best pharmacophore model generated. 


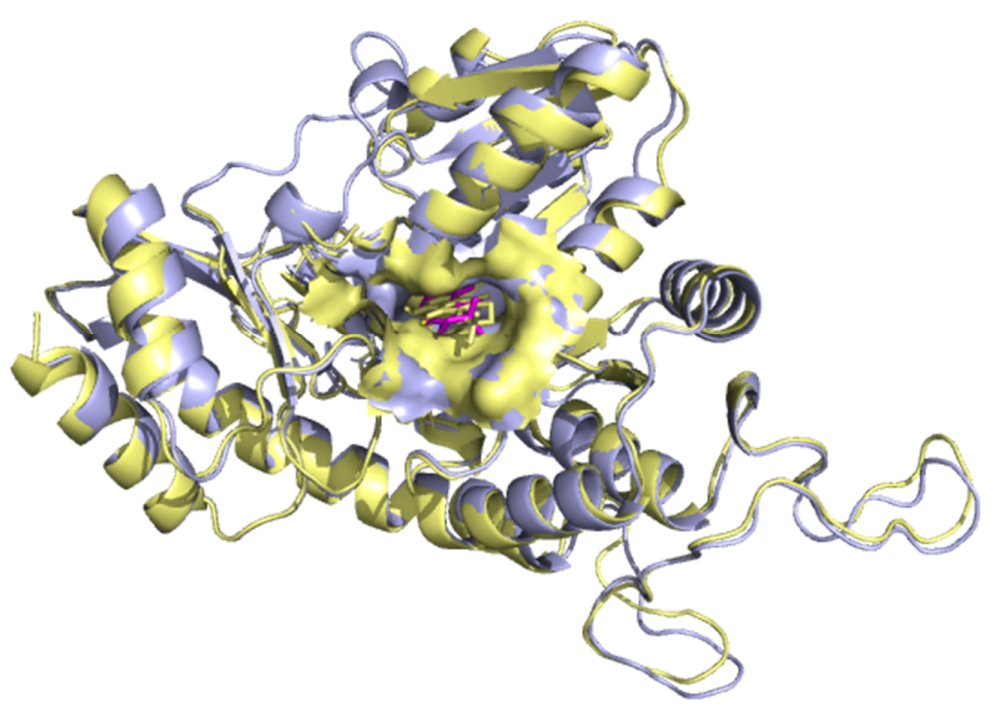

Figure 1. Superimposition of 3qhx (pale yellow) on 4100 (aquamarine). The RMSD for the structural alignment was $0.66 \AA$, which is indicative of the high similarity between the two protein structures.

$\mathbf{A}$
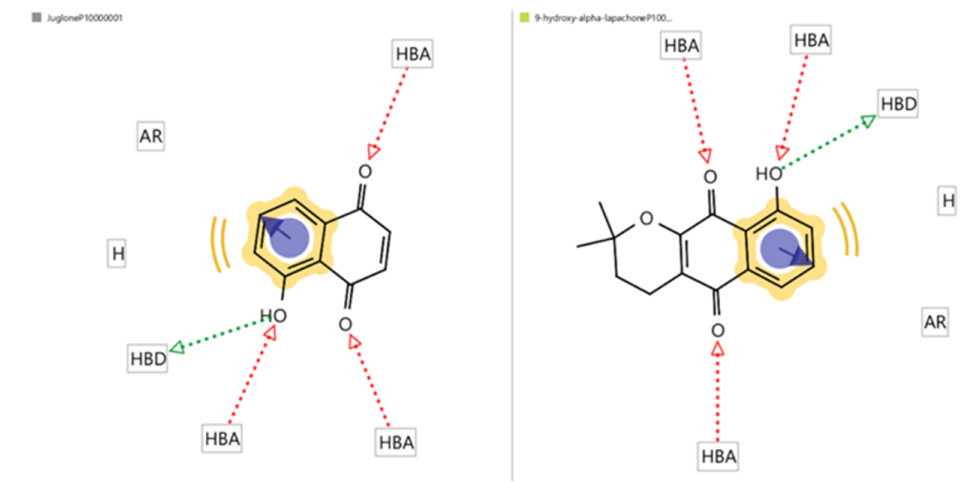

B

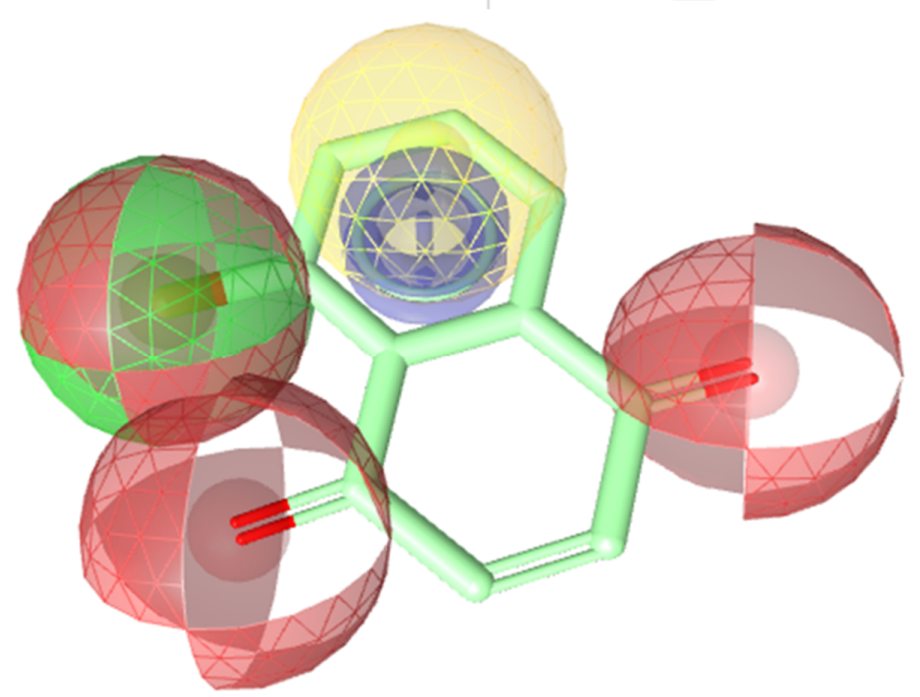

Figure 2. The pharmacophore features generated from the two inhibitors. (A) Juglone (left) and 9-hydroxy-lapachone (right) showing shared pharmacophoric features comprising Aromatic Rings (AR), Hydrogen Bond Donors (HBD), Hydrogen Bond Acceptors (HBA) and Hydrophobic interactions $(\mathrm{H})$. (B) The actives and their overlapped pharmacophoric features which are represented in the pharmacophore model. The red balls show Hydrogen bond acceptors (HBA), the yellow ball shows Hydrophobic interactions, the green ball shows Hydrogen bond donors (HBD) and a blue ring represents an Aromatic ring. 


\subsection{Validation of Generated Pharmacophore Model}

The ROC curve shows the performance of the model to effectively distinguish between a collection of "active" and "inactive" compounds [44]. The AUCs were determined as 1.0, $1.0,1.0$ and 0.70 in the top $1 \%, 5 \%, 10 \%$ and $100 \%$ of the screened library, respectively. AUC is assigned values between 0 and 1 , with 1 suggesting a theoretically perfect classification showing $100 \%$ sensitivity and $100 \%$ specificity. Consequently, an AUC closer to 1 is crucial, whilst 0.5 suggests a random classification implying a poor predictive ability of the model [44-47]. An AUC value of 0 implies an incorrect classification, whilst 0.70 or better implies moderate discrimination and hence suitable for classification. Since the overall AUC was 0.7 , the model was reasonably good in classification. Additionally, EFs were determined as $51.0,34.0,34.0$ and 34.0 for $1 \%, 5 \%, 10 \%$ and $100 \%$, respectively (Figure 3). With three active ligands appearing in the hit results (Figure 3), the selected pharmacophore model had the reasonable capability to distinguish between actives and decoys successfully [48].

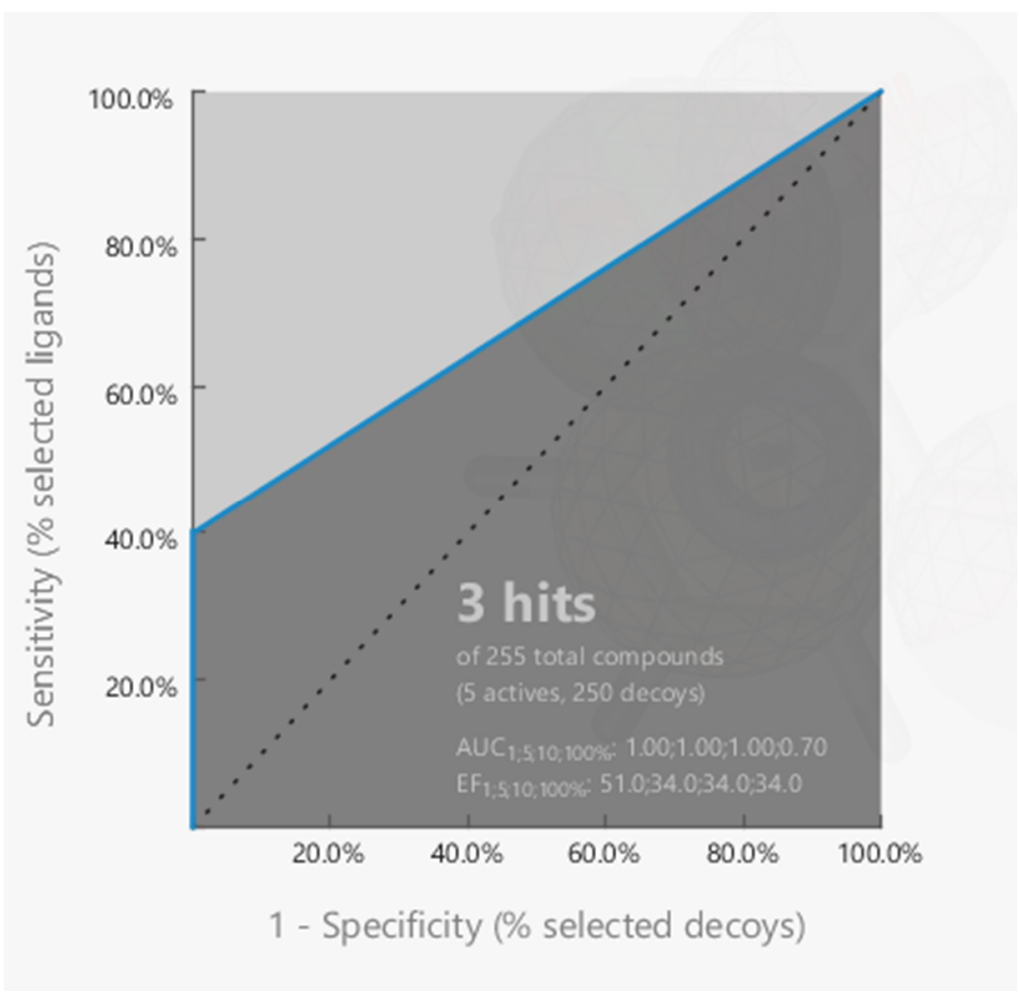

Figure 3. Selected pharmacophore model ROC curve indicated in blue. Determined at 1, 5, 10 and $100 \%$ of the selected database were the AUC and EF values as shown. The median is shown by dotted lines. If the curve were to be close to the median, then it would suggest a poor model.

\subsection{Pharmacophore-Based Screening of the Library}

Virtual screening based on the generation of pharmacophore models is useful in identifying structurally novel and potential lead compounds arising from chemically diverse databases [49]. The validated pharmacophore model was used as a 3D query to screen the library comprising 2530 compounds. Compounds are matched and filtered out based on the pharmacophore fit score generated for the pharmacophore model [26]. This process drastically reduces the dataset of molecules resulting in fewer promising molecules for molecular docking. The compounds used for further downstream analysis had pharmacophore fit scores above 65 (Table 2). The mapping of these compounds on the pharmacophore model is also visualized (Figure 4). 
Table 2. Hit molecules arranged in order of decreasing pharmacophore fit score. The compounds were used for further downstream analysis including molecular docking.

\begin{tabular}{|c|c|}
\hline Name & Pharmacophore-Fit Score \\
\hline Pyrogallol & 67.16 \\
\hline Chrysophanol & 67.04 \\
\hline ZINC00058187 & 67.02 \\
\hline 3-methoxy-4-hydroxyphenol 1-O-beta-D-glucopyranoside & 66.97 \\
\hline Gossypetin 3,7,8-trimethyl ether & 66.88 \\
\hline $3^{\prime}$-hydroxyflindulatin & 66.88 \\
\hline Vanillin & 66.85 \\
\hline $4^{\prime}$-methyl gossypetin & 66.82 \\
\hline Isoscutellarein & 66.81 \\
\hline Corniculatusin & 66.75 \\
\hline Sexangularetin & 66.75 \\
\hline Bucegin & 66.74 \\
\hline Isoscutellarein 8-methyl ether & 66.74 \\
\hline Onopordin & 66.74 \\
\hline Gentisic acid & 66.74 \\
\hline 1,8-dihydroxy-3,5-dimethoxyxanthone & 66.74 \\
\hline Herbacetin & 66.74 \\
\hline ZINC14490611 & 66.72 \\
\hline Vanillic acid & 66.70 \\
\hline Betavulgarin & 66.56 \\
\hline Epitaxifolin & 66.42 \\
\hline P-hydroxybenzoic acid & 66.40 \\
\hline 2,5-dihydroxybenzaldehyde & 66.36 \\
\hline $2,4^{\prime}$-dihydroxy-3'-methoxyacetophenone & 66.35 \\
\hline Omega-hydroxypropioguaiacone & 66.34 \\
\hline 5-(hydroxymethyl)-2-furancarboxylic acid & 66.34 \\
\hline Aloe-emodin & 66.30 \\
\hline Catechin & 66.30 \\
\hline ZINC05854400 & 66.19 \\
\hline 2,5-dihydroxybenzyl alcohol & 66.17 \\
\hline ZINC00013245 & 66.12 \\
\hline Acetovanillone & 66.11 \\
\hline 4-(2-formyl-5-hydroxymethylpyrrol-1-yl) butyric acid & 66.07 \\
\hline Gossypetin 3,8-dimethyl ether & 65.97 \\
\hline 3,7-dihydroxy-8-methoxy-3-(3',4'-methylenedioxybenzyl)chroman-4-one & 65.89 \\
\hline 2,3-dihydroxy-1-(4-hydroxy-3-methoxyphenyl)-1-propanone & 65.88 \\
\hline Shikimic acid-4-O-gallate & 65.85 \\
\hline ZINC13328057 & 65.77 \\
\hline Gentisic acid 5-O-glucoside & 65.44 \\
\hline
\end{tabular}

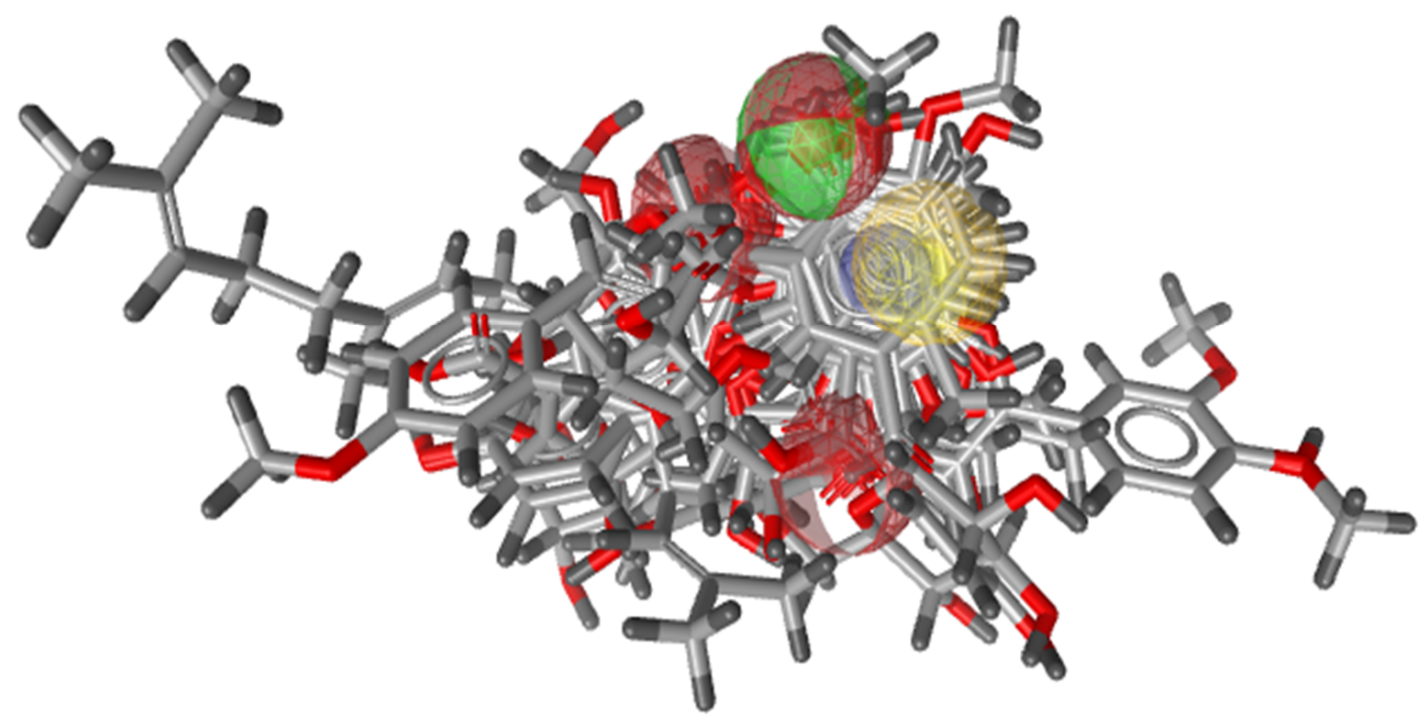

Figure 4. Visualization of hit molecules mapped onto the pharmacophore model. 


\subsection{Validation of Molecular Docking Protocol}

\subsubsection{Superimposition of Co-Crystals with Re-Docked Complexes}

AutoDock Vina was used for molecular docking as a result of its ability to ran on all major operating systems [50]. However, to be used for this study, it needed to be validated. The re-docking of HEPES ligand into the active site was used to evaluate the docking protocol via superimposing the projected docking poses over the experimentally identified co-crystallized structure of the target. The basis of which was the fact that HEPES has a known conformation and orientation since it was extracted from the co-crystal structure of the target. An RMSD value of $1.413 \AA$ was obtained indicating that AutoDock Vina had the potential to reproduce the crystallographic pose. The pose generated from the superimposition of the co-crystallized HEPES and re-docked HEPES complexes is represented in Figure 5. Additionally, four overlapping interacting residues comprising Tyr111, Asn158, Met350, and Arg368 were revealed, which were previously shown as critical (Figure 6). These critical overlapping residues are indicative of the fact that AutoDock Vina could essentially replicate a strikingly comparable pose given the similar setting.

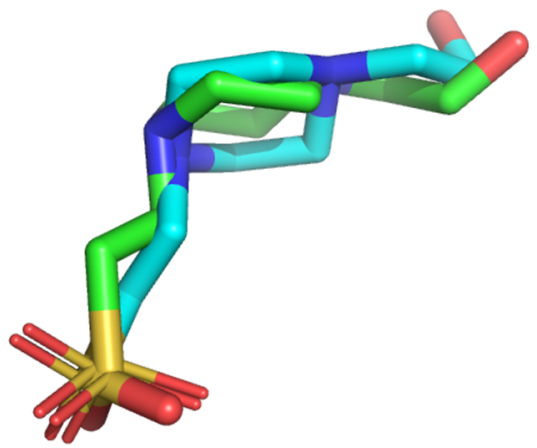

Figure 5. The pose generated for the superimposition of the co-crystallized HEPES molecule (sky blue) and the re-docked HEPES molecule (green). This supports AutoDock Vina as an effective docking protocol.

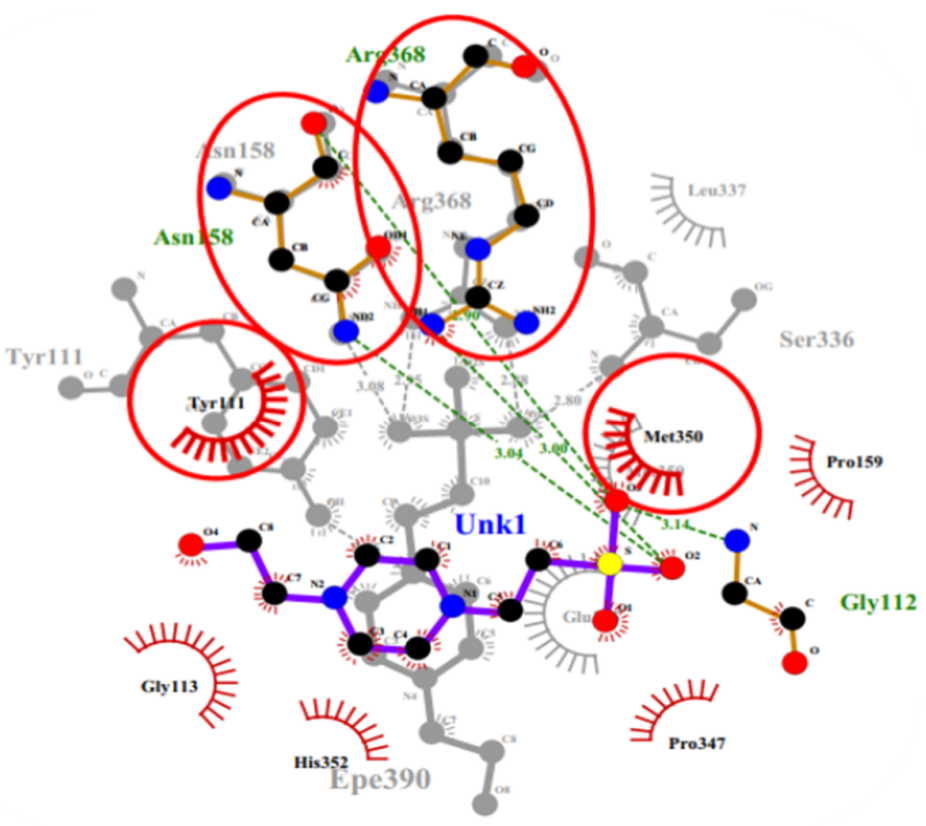

Figure 6. LigPlot+ of superimposition between the co-crystallized ligand of $3 \mathrm{QHX}$ and the re-docked HEPES ligand. Red circles represent the superimposed molecular interactions between both the co-crystallized and the re-docked ligands. 


\subsubsection{ROC Curve Analysis of the Molecular Docking Protocol}

ROC curve with computed AUC is crucial in virtual screening to evaluate the efficiency of AutoDock Vina in its capacity to differentiate between active ligands and inactive molecules or decoys [32]. The AUC of the ROC curve for the five inhibitors of CGS from H. pylori against CGS from M. ulcerans and their corresponding 250 decoys was 0.76 (Figure 7). This suggests that AutoDock Vina showed appreciably good discriminative ability in distinguishing between the 5 inhibitors comprising Juglone, $\alpha$-Lapachone, Paulownin, Yangambin, and 9-Hydroxy- $\alpha$-Lapachone from their corresponding 250 decoys.

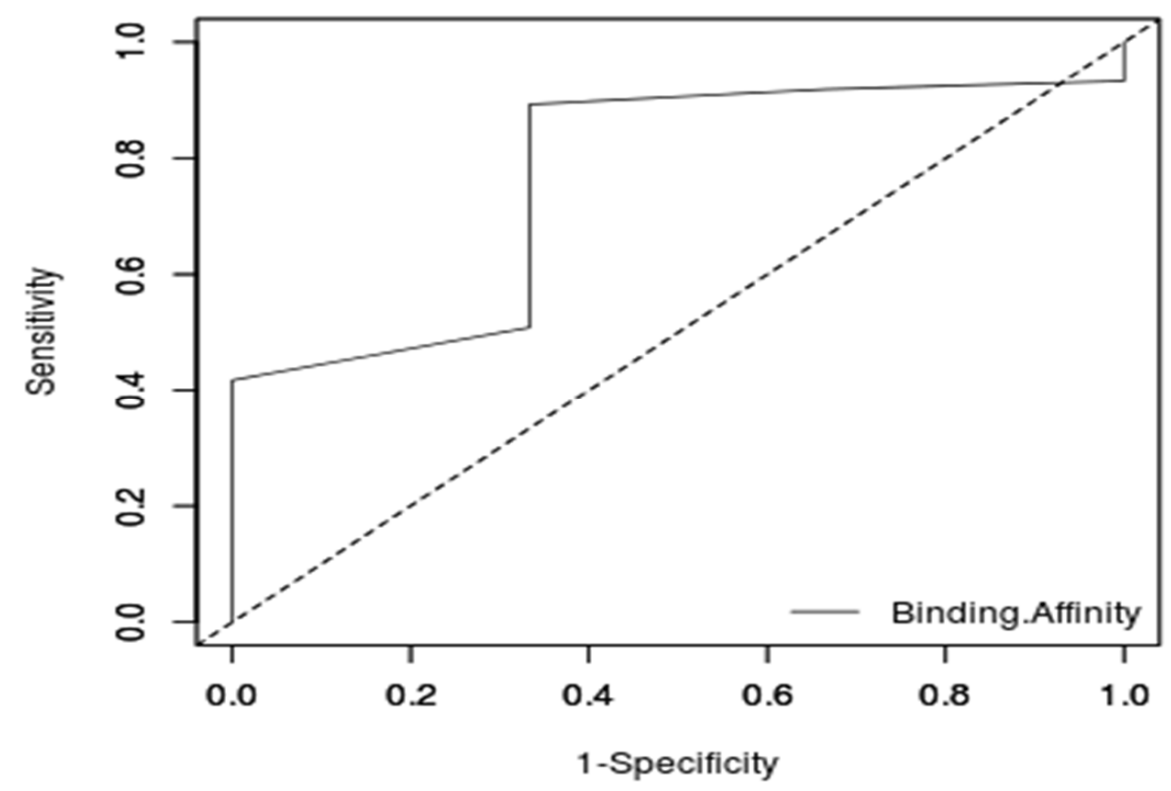

Figure 7. ROC plot generated by screening ligands active against $H$. pylori with their matching decoys against the protein structure, CGS of M. ulcerans. The resulting AUC was 0.76, which was acceptable.

\subsection{Molecular Docking of Pharmacophore Hits}

Molecular docking involves the prediction and identification of ligand orientation and conformation within a targeted active site [51,52]. It involves the identification of lead compounds against a target protein. Molecular docking via virtual screening predicts the ligand-target complex structure by exploring the conformational space of the ligands within the binding site of the target protein. The screened pharmacophore hits (Table 2) were used to obtain ligands with low binding energies (Table 3 and Figure 8). The lower the binding energy, the stronger the binding affinity of the ligand to the target protein. Binding affinity refers to the strength of the interaction between two or more molecules [53]. Table 3 shows the five known inhibitors of CGS of $\mathrm{H}$. pylori and the 24 pharmacophore hits that docked firmly within the binding pocket after filtering out compounds with binding energies $>-7.0 \mathrm{kcal} / \mathrm{mol}$. Chrysophanol reported the lowest binding energy of $-8.9 \mathrm{kcal} / \mathrm{mol}$. The $24 \mathrm{compounds}$ had binding energies within the range of -7.1 to $-8.9 \mathrm{kcal} / \mathrm{mol}$. Similarly, the $5 \mathrm{known}$ inhibitors had binding energies falling within the range of -7.2 to $-8.8 \mathrm{kcal} / \mathrm{mol}$. 
Table 3. Binding energies of top 24 ligands after molecular docking. The ligands with the more negative binding energies are ranked or classified as obtaining the highest binding affinities.

\begin{tabular}{|c|c|c|}
\hline No. & ZINC ID/Compound Name & Binding Energy/kcal/mol \\
\hline \multicolumn{3}{|c|}{ Known Inhibitors } \\
\hline 1 & 9-hydroxy-alpha-lapachone & -8.8 \\
\hline 2 & Alpha-Lapachone & -8.7 \\
\hline 3 & Paulownin & -8.5 \\
\hline 4 & Juglone & -7.3 \\
\hline 5 & Yangambin & -7.2 \\
\hline \multicolumn{3}{|c|}{ Pharmacophore Hits } \\
\hline 6 & Chrysophanol & -8.9 \\
\hline 7 & Aloe-emodin & -8.6 \\
\hline 8 & Herbacetin & -8.5 \\
\hline 9 & Isoscutellarein & -8.4 \\
\hline 10 & Onopordin & -8.4 \\
\hline 11 & Betavulgarin & -8.4 \\
\hline 12 & ZINC05854400 & -8.4 \\
\hline 13 & ZINC14490611 & -8.3 \\
\hline 14 & Bucegin & -8.3 \\
\hline 15 & Isoscutellarein 8-methyl ether & -8.2 \\
\hline 16 & Sexangularetin & -8.2 \\
\hline 17 & Corniculatusin & -8.2 \\
\hline 18 & $4^{\prime}$-methyl gossypetin & -8.2 \\
\hline 19 & 1,8-dihydroxy-3,5-dimethoxyxanthone & -8.1 \\
\hline 20 & Epitaxifolin & -8.1 \\
\hline 21 & ZINC13328057 & -7.9 \\
\hline 22 & Catechin & -7.7 \\
\hline 23 & Gossypetin 3,8-dimethyl ether & -7.7 \\
\hline 24 & Gossypetin 3,7,8-trimethyl ether & -7.7 \\
\hline 25 & ZINCO0058187 & -7.6 \\
\hline 26 & Shikimic acid-4-O-gallate & -7.6 \\
\hline 27 & $\begin{array}{l}\text { 3,7-dihydroxy-8-methoxy-3- }\left(3^{\prime}, 4^{\prime}-\right. \\
\text { methylenedioxybenzyl)chroman-4-one }\end{array}$ & -7.6 \\
\hline 28 & 3'-hydroxyflindulatin & -7.6 \\
\hline 29 & Gentisic acid 5-O-glucoside & -7.1 \\
\hline
\end{tabular}

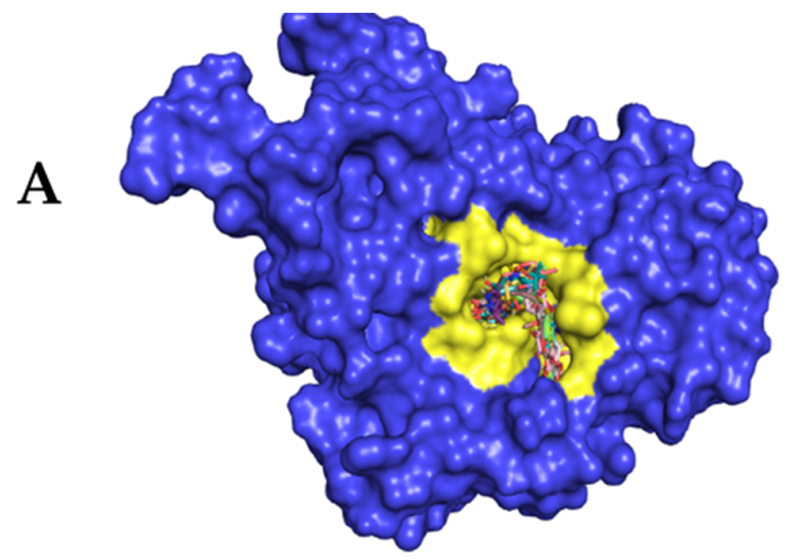

B

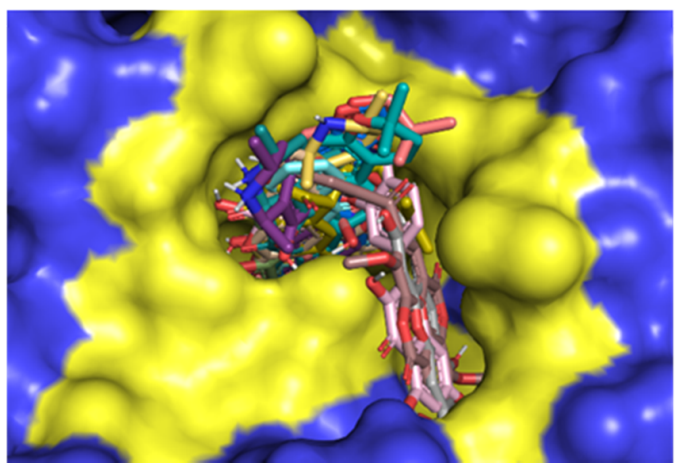

Figure 8. (A) Surface representation of ligand-target complex showing the docking results of best 24 hit ligands in the active site region colored cyan. (B) Magnified view showing ligands docked firmly in the pocket. 


\subsection{Protein-Ligand Interaction}

Proteins are known to attain their optimum biological functions by their direct physical interaction with ligands which aids in a better understanding of protein functions and drug development [54]. Hydrogen bonding is one of the most essential intermolecular interactions because it confers much stability to the protein-ligand complex [55-57]. The ligands of interest are those that formed more hydrogen bond interactions with the protein, demonstrating potential specificity which would distinguish a highly specific binding partner from less specific ones [58].

The highest number of hydrogen bonds of six was formed among Shikimic acid4-O-gallate and the receptor. Epitaxilon and Gentisic acid 5-O glucoside followed with 5 hydrogen bonds each (Figure 9) and then Betavulgarin with 4 hydrogen bond interactions. The exception was Catechin which formed no hydrogen bonds with the receptor. Juglone and 9-hydroxy-alpha-lapachone were found to have formed 2 and 1 hydrogen bonds with the Asp183 residue, respectively.

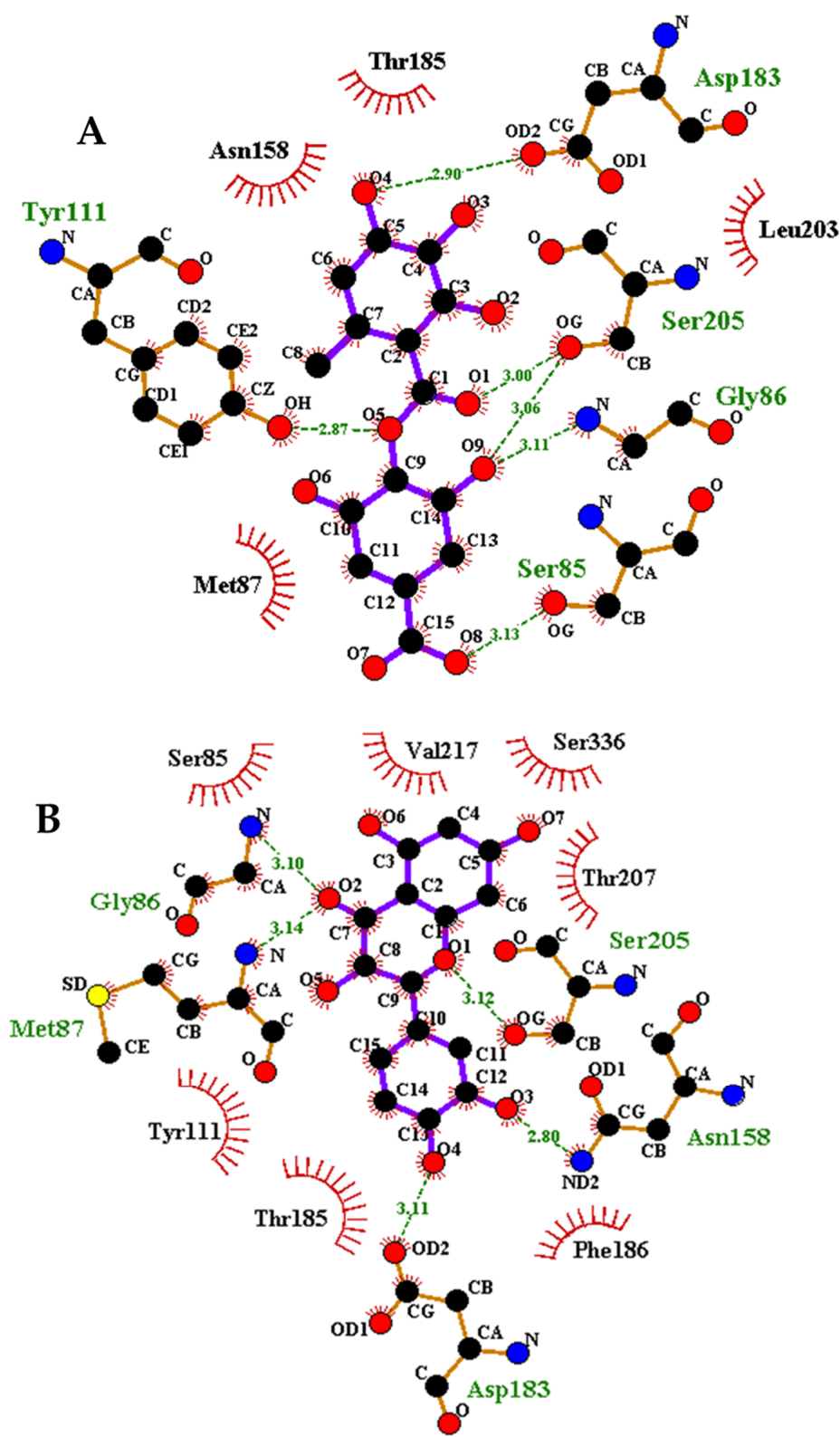

Figure 9. Cont. 


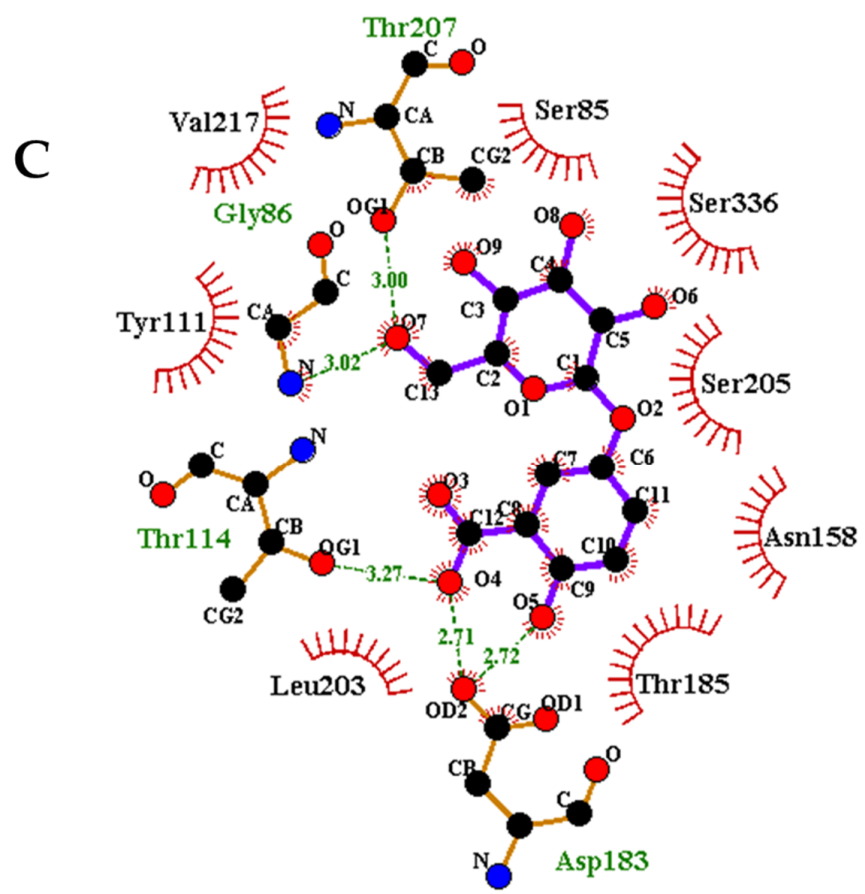

Figure 9. Protein-ligand interactions elucidated with LigPlot+. Shikimic acid-4-O-gallate (A), Epitaxifolin (B), and Gentisic acid 5-O-glucoside (C) represented by blue sticks. Carbon atoms are signified as black dots and red dots show oxygen atoms. Hydrophobic contact residues with the ligands are shown as semi-circles with "spokes". Hydrogen bond interaction patterns are represented as green dash lines.

The shorter the bond length, the stronger the hydrogen bond formed [55]. The shortest hydrogen bond length observed was $2.28 \AA$, which was formed between $4^{\prime}$-methyl gossypetin and Asp183 residue. A summary of the interaction studies of the top 24 hits together with the five known inhibitors is presented in Table S1.

\subsection{Physicochemical Profiling}

None of the 24 ligands violated Lipinski's rule since pre-filtering of the library was undertaken to eliminate all potential "non-drug-like" compounds. Similarly, the known inhibitors violated none of Lipinski's rules. Other physicochemical parameters predicted have been reported (Tables S2 and S3). All 24 ligands showed good physicochemical properties based on the parameters set. When compared with four already existing drugs comprising Streptomycin, Ciprofloxacin, Rifampicin, and Clarithromycin, the hits were predicted to show better physicochemical properties.

\subsection{Pharmacokinetics and Toxicity Studies}

Pharmacokinetics determines the fate of the administered drugs in a living organism after chemical metabolism until elimination from the body [59]. The parameters measured were blood-brain barrier (BBB) permeation, gastrointestinal absorption, permeability glycoprotein, and cytochromes P450 (CYP) (Figure S1 and Table S3). Gastrointestinal absorption (GI) is the process where orally administered drugs are absorbed into the bloodstream [60]. Compounds denoted "high" have a high GI absorption and vice versa. BBB permeation is the potential of a drug to cross the blood-brain barrier to the brain to bind to receptors relevant for the activation of signaling pathways. Predicting the permeability is very important in the development of drugs because a molecule will not be able to demonstrate therapeutic activity within the brain parenchyma unless the barrier is permeated [61]. However, for this study, compounds that are known to cross the BBB were ignored. P-glycoprotein (Pgp) helps to prevent the central nervous system (CNS) from xenobiotics. Cytochrome isoforms CYP1A2, CYP2C9, CYP2C19, CYP2D6, and CYP3A4 are 
essential because of their interaction with compounds to aid in drug elimination through metabolism. Toxic and unwanted adverse effects may occur if these isoenzymes are inhibited. Figure S1 shows the pharmacokinetics of the ligands compared to the existing anti-Buruli ulcer drugs. Compounds are considered to have met the pharmacokinetic threshold if they have high GI absorption, 'No' for BBB permeation, 'Yes' Pgp substrate, and ' $\mathrm{No}^{\prime}$ ' for at least 3 cytochromes.

Aloe-emodin, ZINC05854400, ZINC14490611, Epitaxifolin, ZINC13328057, and Catechin showed good ADME profiles. Shikimic acid-4-O-gallate and Gentisic acid 5-Oglucoside also showed good ADME profiles except for low GI absorption. The rest of the compounds showed poor ADME profiles which suggest that these compounds could be optimized to meet the aforementioned criteria for a good ADME profile. For the known drugs comprising Streptomycin, Ciprofloxacin, Rifampicin, and Clarithromycin, all but Ciprofloxacin showed low GI. All known drugs were predicted to be Pgp substrates. CYP3A4 was the only cytochrome predicted to be inhibited by Clarithromycin. The other known drugs showed no inhibition against the cytochrome family. Salmonella typhimurium reverse mutation assay (AMES) toxicity [62,63], carcinogenicity and Human Ether-a-go-goRelated Gene Inhibition (hERG I Inhibitor) [64] were employed for toxicity studies. AMES toxicity provides information on whether a particular chemical can cause mutations in the DNA of an organism. This was very essential to this study because administered drugs should not cause mutation in the DNA of patients. Carcinogenicity is the measure of a chemical's ability to induce cancer or increase the incidence of cancer, whilst the hERG I Inhibitor represents the potential of the compound to inhibit the hERG I receptor resulting in arrhythmia [65]. For this study, we required that these parameters were adequately met for the compounds to be considered as potentially non-toxic. Table S4 shows the toxicity measure of each ligand as well as known inhibitors. For a ligand to be considered as potentially toxic, it is assigned a value of 1 , and 0 if non-toxic. Nine ligands were found to be AMES toxic. Juglone was the only inhibitor found to be AMES toxic. Additionally, 3 '-hydroxyflindulatin was predicted to be an hERG I inhibitor. Even though it was observed not to be carcinogenic and AMES toxic, it was still classified as potentially toxic. All 24 compounds and 5 inhibitors were found not to be carcinogenic. All compounds that were predicted to be toxic could be adequately optimized to generate low toxic analogues.

\subsection{Exploring Predicted Leads for Anti-Microbial and Antimycobacterial Activity.}

Structure-Activity Relationship (SAR) describes the association between a chemical compound and the intrinsic property of a compound to elicit a particular pharmacological effect [66]. PASS predictions are built on SAR analysis of compounds in the database. In essence, the structural features of queried compounds are compared to those of known biological activity to infer the pharmacological profiles $[67,68]$. Each activity predicted has accompanying Probability of activity (Pa) and Probability of inactivity (Pi) values that determine the probability of a particular substance belonging to a class of active or inactive compounds, respectively. For this study, compounds with $\mathrm{Pa}>\mathrm{Pi}$ for antibacterial and antimycobacterial activities were of particular interest since they are considered probable [68]. A total of 23 compounds were predicted as possessing antibacterial activity with $\mathrm{Pa}>0.3$. Only 3, 7-dihydroxy-8-methoxy-3-( $3^{\prime}, 4^{\prime}$-methylenedioxybenzyl) chroman-4-one was not predicted as antibacterial, hence it was eliminated from the prioritization. Gentisic acid 5-O-glucoside was found to have the highest $\mathrm{Pa} 0.618$ and lowest $\mathrm{Pi} 0.008$. The Pa values for the remaining 22 compounds ranged between $0.3<\mathrm{Pa}<0.6$. Additionally, four of the compounds were in the range $0.4<\mathrm{Pa}<0.5$, while the rest of the 17 were within the range $0.3<\mathrm{Pa}<0.4$ (Table 4 ). 
Table 4. Compounds with their $\mathrm{Pa}$ and Pi values for predicted antibacterial activity. When $\mathrm{Pa}>\mathrm{Pi}$, the compound is worth being pharmacologically profiled.

\begin{tabular}{ccc}
\hline Compounds & $\mathbf{P a}$ & $\mathbf{P i}$ \\
\hline Gentisic acid 5-O-glucoside & 0.618 & 0.008 \\
ZINC05854400 & 0.489 & 0.017 \\
ZINC00058187 & 0.411 & 0.027 \\
Sexangularetin & 0.405 & 0.029 \\
Isoscutellarein 8-methyl ether & 0.404 & 0.029 \\
Isoscutellarein & 0.403 & 0.029 \\
Herbacetin & 0.399 & 0.030 \\
Onopordin & 0.396 & 0.031 \\
Bucegin & 0.394 & 0.031 \\
Gossypetin 3,8-dimethyl ether & 0.393 & 0.032 \\
Gossypetin 3,7,8-trimethyl ether & 0.384 & 0.034 \\
3'-hydroxyflindulatin & 0.384 & 0.034 \\
Epitaxifolin & 0.381 & 0.035 \\
ZINC14490611 & 0.379 & 0.035 \\
Corniculatusin & 0.379 & 0.035 \\
$4^{\prime}$-methyl gossypetin & 0.373 & 0.037 \\
Chrysophanol & 0.371 & 0.038 \\
Aloe-emodin & 0.368 & 0.038 \\
ZINC13328057 & 0.360 & 0.040 \\
Betavulgarin & 0.358 & 0.041 \\
Catechin & 0.354 & 0.042 \\
1,8-dihydroxy-3,-dimethoxyxanthone & 0.350 & 0.043 \\
\hline
\end{tabular}

Medicinal plants that have been suggested to hold inhibitory potential against $M$. ulcerans were found to have Chrysophanol, Chrysophanic acid and aloe-emodin as part of the main constituents. More so, glucoside, methyl ether and gallate functional groups were also found to be the main components of the plants with inhibitory potential against M. ulcerans [69]. Therefore, the compounds could hold inhibitory potential against CGS of M. ulcerans. Six compounds comprising Gentisic acid 5-O-glucoside, ZINC05854400, ZINC00058187, Sexangularetin, Isoscutellarein 8-methyl ether and Isoscutellarein were predicted as antibacterial with $\mathrm{Pa}>0.4$ and $\mathrm{Pa}>\mathrm{Pi}$. Additionally, the $\mathrm{Pa}$ for antimycobacterial, anti-ulcerative, antituberculosis, antioxidant and dermatological activities were within the ranges $0.4<\mathrm{Pa}<0.7,0.3<\mathrm{Pa}<0.7,0.4<\mathrm{Pa}<0.6,0.3<\mathrm{Pa}<0.9$ and $0.3<\mathrm{Pa}<0.6$, respectively. The compounds had $\mathrm{Pa}>\mathrm{Pi}$ for all the predicted biological activities.

Gentisic acid 5-O-glucoside was predicted as antimycobacterial, anti-ulcerative, antituberculosis, antioxidant and dermatological with Pa 0.623 and Pi 0.009, Pa 0.550 and Pi 0.16, $\mathrm{Pa} 0.548$ and Pi 0.008, Pa 0.637 and Pi 0.004, and Pa 0.457 and Pi 0.041, respectively (Table 5). Additionally, Gentisic acid 5-O-glucoside was predicted to be an isocitrate lyase inhibitor with Pa 0.573 and Pi 0.003. Isocitrate lyase (ICL) and maltase synthase (MS) are two essential enzymes in the glyoxylate cycle, a pathway essential to the growth of bacteria [70]. This pathway mediates the persistence of $M$. tuberculosis because of the intermediates of the tricarboxylic acid (TCA) made available to the organism for glucogenesis as well as other biosynthetic processes. Isocitrate lyase has been suggested as a drug target due to its role in disrupting the pathway when it undergoes conformational changes after binding to a substrate [71]. Therefore, Gentisic acid 5-O-glucoside is an attractive molecule to be explored pharmacologically and potentially specific for the target (CGS). Gentisic acid 5-O-glucoside formed a total of 13 intermolecular bonds comprising five hydrogen and eight hydrophobic bonds.

A similarity search through DrugBank revealed Quercetin and Diosmetin as analogs of Isoscutellarein with a similarity score of 0.838 each. Quercetin and Diosmetin have been linked to $M$. tuberculosis as possessing antimycobacterial and antituberculosis potentials [72-74]. Quercetin and Diosmetin belong to the family of flavonoids which are known 
to be principal constituents of plants with potency against M. ulcerans [69]. Isoscutellarein had a binding energy value of $-8.4 \mathrm{kcal} / \mathrm{mol}$ and formed 3 hydrogen bond interactions with critical residues Met87, Asp183 and Glu154, as well as 9 hydrophobic contacts.

A structural similarity search via DrugBank with a similarity score of 0.7 involving ZINC05854400 and ZINC00058187 did not reveal any analogs reported to be antimycobacterial or antibacterial. However, when the threshold was decreased further, Quercetin and Atovaquone emerged with similarity scores of 0.582 and 0.553 for ZINC05854400 and ZINC00058187, respectively. A combination of Atovaquone and Azithromycin has been shown as a viable therapy for Mycobacterium avium complex (MAC) infection [75]. Furthermore, ZINC05854400 and ZINC00058187 were among the three compounds (Table 5) predicted as dermatological with Pa $>0.3$. ZINC05854400 had a binding energy of $-8.4 \mathrm{kcal} / \mathrm{mol}$ and formed 2 hydrogen bond interactions with two critical residues Met87 and Asp183, as well as 12 hydrophobic contacts. ZINC00058187 had a binding energy of $-7.6 \mathrm{kcal} / \mathrm{mol}$ and formed 1 hydrogen bond interaction with Asn158, as well as 7 hydrophobic contacts.

Table 5. Predicted biological activities of compounds with $\mathrm{Pa}>0.3$ and $\mathrm{Pi}<\mathrm{Pa}$. The biological activities comprise antimycobacterial, anti-ulcerative, antituberculosis, antioxidant and dermatological activity.

\begin{tabular}{|c|c|c|c|c|c|}
\hline \multicolumn{3}{|c|}{ Antimycobacterial } & \multicolumn{3}{|c|}{ Anti-Ulcerative } \\
\hline Compounds & $\mathbf{P a}$ & $\mathbf{P i}$ & Compounds & $\mathbf{P a}$ & $\mathbf{P i}$ \\
\hline $\begin{array}{l}\text { Gentisic acid } \\
\text { 5-O-glucoside }\end{array}$ & 0.623 & 0.009 & ZINC05854400 & 0.637 & 0.008 \\
\hline $\begin{array}{l}\text { Isoscutellarein } \\
\text { 8-methyl ether }\end{array}$ & 0.568 & 0.012 & $\begin{array}{l}\text { Gentisic acid } \\
\text { 5-O-glucoside }\end{array}$ & 0.550 & 0.016 \\
\hline ZINC00058187 & 0.51 & 0.018 & Isoscutellarein & 0.536 & 0.017 \\
\hline Sexangularetin & 0.47 & 0.024 & $\begin{array}{l}\text { Isoscutellarein } \\
\text { 8-methyl ether }\end{array}$ & 0.521 & 0.02 \\
\hline Isoscutellarein & 0.465 & 0.025 & Sexangularetin & 0.486 & 0.026 \\
\hline ZINC05854400 & 0.446 & 0.029 & ZINC00058187 & 0.333 & 0.076 \\
\hline \multicolumn{3}{|c|}{ Antituberculosis } & \multicolumn{3}{|c|}{ Antioxidant } \\
\hline Compounds & $\mathrm{Pa}$ & $\mathbf{P i}$ & Compounds & $\mathbf{P a}$ & $\mathbf{P i}$ \\
\hline $\begin{array}{l}\text { Gentisic acid } \\
\text { 5-O-glucoside }\end{array}$ & 0.548 & 0.008 & Isoscutellarein & 0.876 & 0.003 \\
\hline $\begin{array}{l}\text { Isoscutellarein } \\
\text { 8-methyl ether }\end{array}$ & 0.502 & 0.02 & ZINC05854400 & 0.837 & 0.003 \\
\hline ZINC00058187 & 0.492 & 0.013 & Sexangularetin & 0.825 & 0.003 \\
\hline Sexangularetin & 0.443 & 0.021 & $\begin{array}{l}\text { Isoscutellarein } \\
\text { 8-methyl ether }\end{array}$ & 0.777 & 0.004 \\
\hline Isoscutellarein & 0.438 & 0.022 & $\begin{array}{l}\text { Gentisic acid } \\
5 \text {-O-glucoside }\end{array}$ & 0.637 & 0.004 \\
\hline \multicolumn{3}{|c|}{ Dermatological } & ZINC00058187 & 0.343 & 0.017 \\
\hline Compounds & $\mathrm{Pa}$ & $\mathbf{P i}$ & & & \\
\hline ZINC05854400 & 0.501 & 0.032 & & & \\
\hline $\begin{array}{l}\text { Gentisic acid } \\
5 \text {-O-glucoside }\end{array}$ & 0.457 & 0.041 & & & \\
\hline ZINC00058187 & 0.356 & 0.065 & & & \\
\hline
\end{tabular}

\subsection{Molecular Dynamics (MD) Simulation of Target Structure and Complexes}

MD simulation was executed to study the stability and conformational changes of the target protein and ligands in complex with the protein. Five receptor-ligand complexes and the unbound receptor were subjected to MD simulations. The ligands comprise two known inhibitors Juglone and 9-hydroxy alpha-lapachone and potential leads Gentisic acid 5-O glucoside, Isoscutellarein, and ZINC05854400. The stabilities were analyzed over $100 \mathrm{~ns}$ simulation time (Figure 10). The RMSD plots were used to assess the stability of 
the complexes and the target protein. The RMSD represents the average distance of atoms of the residues at the protein backbone [76]. The backbone of the target protein showed fluctuations from $0.15 \mathrm{~nm}$ to $0.25 \mathrm{~nm}$ over the initial period of 5 . However, it sustained an average RMSD of $0.25 \mathrm{~nm}$ from $5 \mathrm{~ns}$ to about $55 \mathrm{~ns}$, then fluctuated to about $3.0 \mathrm{~nm}$, where it remained steady until $100 \mathrm{~ns}$ period, albeit, recording an RMSD of $0.27 \mathrm{~nm}$. Aside from the initial fluctuations between $0.25 \mathrm{~nm}$ and $0.35 \mathrm{~nm}$ from 0 to $30 \mathrm{~ns}$, both Isoscutellarein and Gentisic 5-O glucoside complexes mimicked the trajectory of the target protein until the $100 \mathrm{~ns}$ period, attaining an average RMSD of about $0.27 \mathrm{~nm}$ in both cases. The RMSD of the ZINC 05854400 complex rose to about $0.44 \mathrm{~nm}$ for the initial $20 \mathrm{~ns}$ and remained steady until few fluctuations at the $65 \mathrm{~ns}$ and then rose to $0.41 \mathrm{~nm}$ at $75 \mathrm{~ns}$, where it remained steady until the $100 \mathrm{~ns}$ period, averaging at RMSD of about $0.42 \mathrm{~nm}$. The RMSD of the 9-hydroxy alpha-lapachone complex rose to about $0.45 \mathrm{~nm}$ for the initial $20 \mathrm{~ns}$ and progressed steadily until $50 \mathrm{~ns}$ where it dropped to about $0.28 \mathrm{~ns}$. It increased again to about $0.41 \mathrm{~nm}$ with few fluctuations between $0.35 \mathrm{~nm}$ to $0.45 \mathrm{~nm}$ towards the $100 \mathrm{~ns}$ mark. The RMSD of the Juglone complex rose to about $0.4 \mathrm{~nm}$ at $5 \mathrm{~ns}$ and remained steady to about $53 \mathrm{~ns}$ where it increased for about $0.45 \mathrm{~ns}$. It then remained steady until $86 \mathrm{~ns}$ where it rose to about 0.52 ns until 100 ns. Therefore, the complexes were conformationally stable within the period of the simulations.

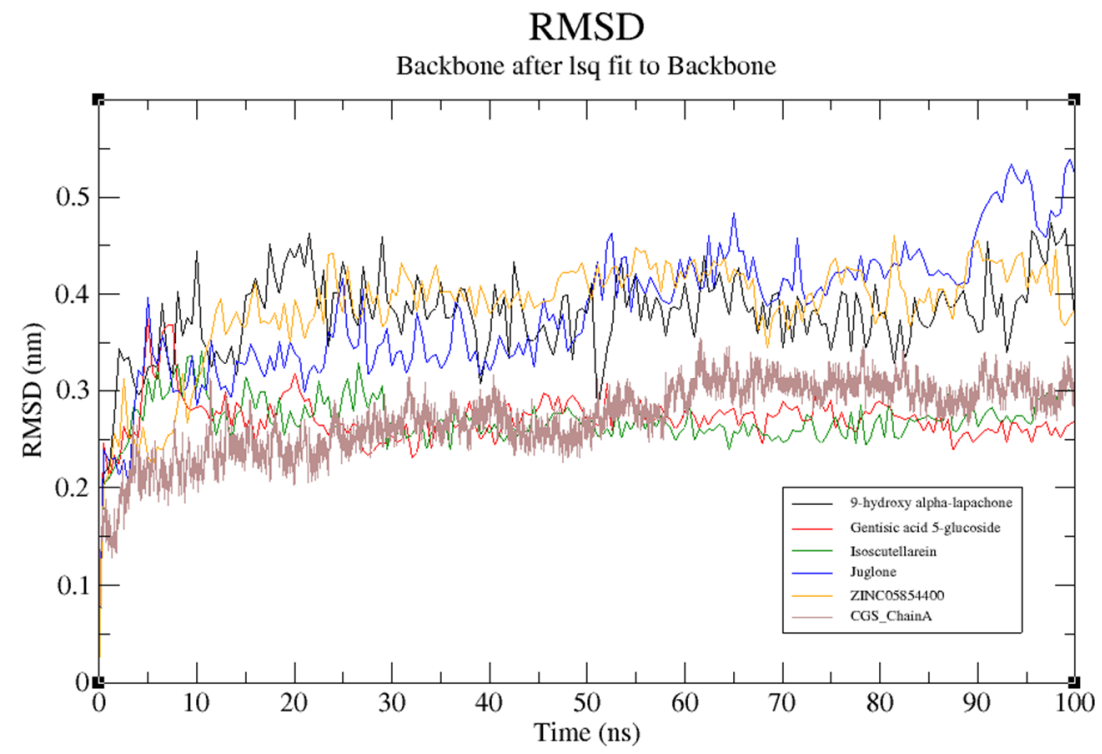

Figure 10. RMSD versus time plot of the backbone of CGS superimposed with CGS complexes. The molecular dynamics simulations were run for $100 \mathrm{~ns}$.

\subsection{MM-PBSA Binding Free Energy Calculations}

The MM-PBSA approach [32] was used in calculating the binding free energies of the protein-ligand complexes following the contribution of van der Waal, electrostatic, polar solvation, and the Solvent Accessible Surface Area (SASA) energies [77]. Gentisic 5-O glucoside obtained the least free binding energy of $-239.865 \mathrm{~kJ} / \mathrm{mol}$ (Table 6) and was followed by Isoscutellarein with $-70.790 \mathrm{~kJ} / \mathrm{mol}$. Even though ZINC05854400 was predicted to have the least affinity to the protein with a binding free energy of $320.907 \mathrm{~kJ} / \mathrm{mol}$, it was found to have one of the lowest binding energies of $-8.4 \mathrm{kcal} / \mathrm{mol}$ from the molecular docking. In terms of energy contribution to the overall binding energy, van der Waal had a larger energy contribution whereas nonpolar solvation energies and electrostatic energies contributed slightly (Table 6).

Per-residue decomposition was employed to investigate the energy input of each residue. In general, residues with energies $>5.0 \mathrm{~kJ} / \mathrm{mol}$ or $<-5.0 \mathrm{~kJ} / \mathrm{mol}$ are regarded as plausible key binding moieties [78]. Majority of the residues contributed energies $>5.0 \mathrm{~kJ} / \mathrm{mol}$ or $<-5.0 \mathrm{~kJ} / \mathrm{mol}$ in both Gentisic 5-O glucoside (Figure S2) and ZINC05854400 complexes 
(Figure 11). Isoscutellarein complex (Figure S3) on the other hand had just one residue, Leu181, with energy contribution $>5.0 \mathrm{~kJ} / \mathrm{mol}$. Even though, Gentisic $5-\mathrm{O}$ glucoside and ZINC05854400 complexes were hitherto reported to bind to crucial residues (Gly86, Met87, Asn158, Asp183, and Ser205), only Met87 produced the highest energy contribution (Figure 11). Even though, other residues contributed more, they were not located in the binding pocket of the protein.

Table 6. Energy terms of the CGS-ligand complexes from MM-PBSA calculation. The values are presented in average \pm standard deviations in $\mathrm{kJ} / \mathrm{mol}$.

\begin{tabular}{cccccc}
\hline Compound & $\begin{array}{c}\text { van der Waal } \\
\text { Energy (KJ/mol) }\end{array}$ & $\begin{array}{c}\text { Electrostatic } \\
\text { Energy (KJ/mol) }\end{array}$ & $\begin{array}{c}\text { Polar Solvation } \\
\text { Energy (KJ/mol) }\end{array}$ & $\begin{array}{c}\text { SASA Energy } \\
(\mathbf{K J} / \mathbf{m o l})\end{array}$ & $\begin{array}{c}\text { Binding Energy } \\
\mathbf{( K J} / \mathbf{m o l})\end{array}$ \\
\hline Gentisic 5-O & $1.793+/-$ & $-633.686+/-$ & $398.347+/-$ & $-6.320+/-$ & $-239.865+/-$ \\
glucoside & $6.832 \mathrm{~kJ} / \mathrm{mol}$ & $68.712 \mathrm{~kJ} / \mathrm{mol}$ & $34.000 \mathrm{~kJ} / \mathrm{mol}$ & $2.460 \mathrm{~kJ} / \mathrm{mol}$ & $68.428 \mathrm{~kJ} / \mathrm{mol}$ \\
& $-101.940+/-$ & $-12.961+/-$ & $54.056+/-$ & $-9.944+/-$ & $-70.790+/-$ \\
Isoscutellarein & $79.145 \mathrm{~kJ} / \mathrm{mol}$ & $12.615 \mathrm{~kJ} / \mathrm{mol}$ & $51.199 \mathrm{~kJ} / \mathrm{mol}$ & $8.391 \mathrm{~kJ} / \mathrm{mol}$ & $60.419 \mathrm{~kJ} / \mathrm{mol}$ \\
& $-117.075+/-$ & $356.619+/-$ & $95.183+/-$ & $-13.820+/-$ & $320.907+/-$ \\
ZINC05854400 & $30.579 \mathrm{~kJ} / \mathrm{mol}$ & $91.497 \mathrm{~kJ} / \mathrm{mol}$ & $77.435 \mathrm{~kJ} / \mathrm{mol}$ & $3.671 \mathrm{~kJ} / \mathrm{mol}$ & $54.474 \mathrm{~kJ} / \mathrm{mol}$ \\
\hline
\end{tabular}

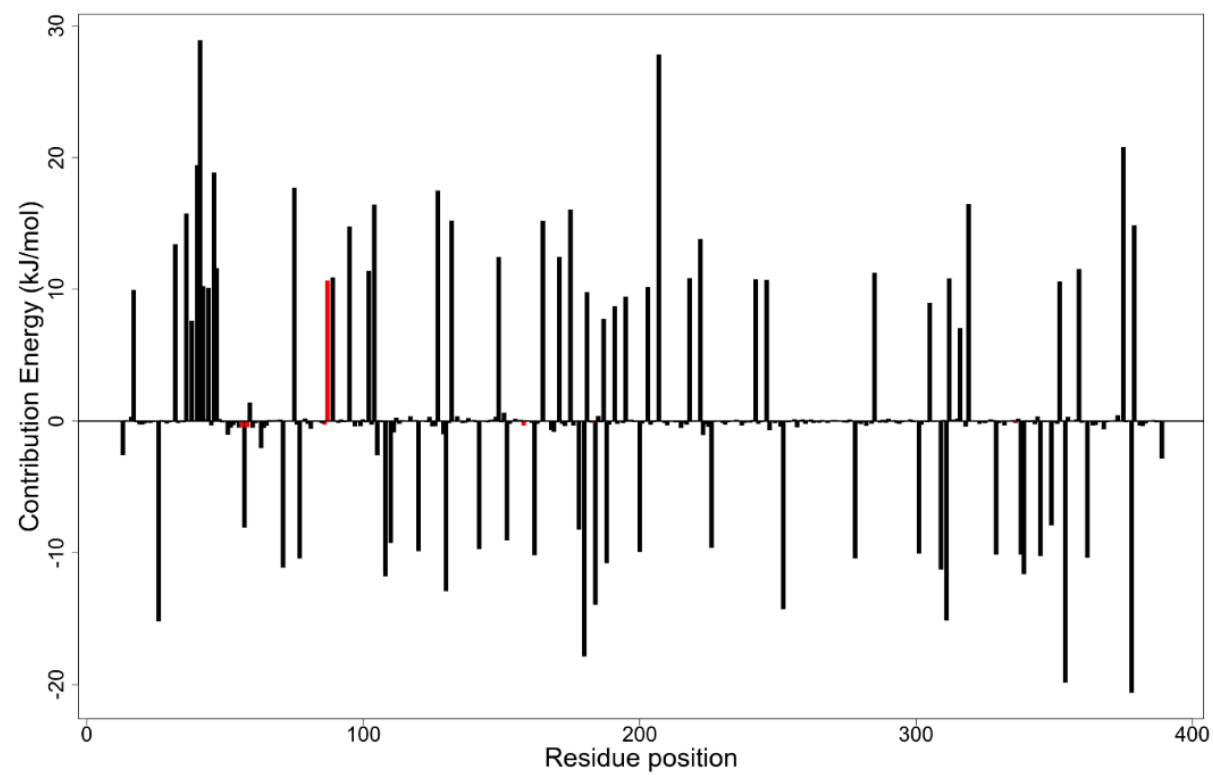

Figure 11. MM-PBSA plot of ZINC05854400 complex showing binding free energy contribution per-residue.

\subsection{Leads Summary}

Gentisic acid 5-O glucoside, Isoscutellarein and ZINC05854400 have been identified as potential leads against CGS of M. ulcerans (Table 7). Even though, ZINC05854400 had the highest binding free energy of $320.907 \mathrm{~kJ} / \mathrm{mol}$ with CGS of M. ulcerans from the MM-PBSA calculations, its antimycobacterial potential can be explored experimentally. However, it could be exploited as a scaffold for the development of antimycobacterial drugs. The antimycobacterial potential of the compounds Sexangularetin, Isoscutellarein 8-methyl ether, and ZINC00058187 could also be further investigated. 
Table 7. Molecules with their names and 2D structures. The molecules have been suggested as potential antimycobacterial leads.

Gentisic acid 5-O glucoside

Isoscutellarein

ZINC05854400 2-hydroxy-5-[3,4,5-trihydroxy-6-

(hydroxymethyl)oxan-2-yl]oxybenzoic acid 5,7,8-trihydroxy-2-

(4-hydroxyphenyl)chromen-4-one<smiles>O=C(O)c1cc(OC2OC(CO)C(O)C(O)C2O)ccc1O</smiles><smiles>O=c1cc(-c2ccc(O)cc2)oc2c(Cl)c(Cl)cc(O)c12</smiles><smiles>CC(C)=CCc1c(O)c(CC=C(C)C)c2oc3c(O)ccc(O)c3c(=O)c2c1O</smiles>

\section{Conclusions}

We have used molecular docking and ligand-based pharmacophore modeling to identify three African natural products as potential novel lead compounds against the CGS of M. ulcerans. The compounds comprising Gentisic acid 5-O glucoside, Isoscutellarein and ZINC05854400 have the potential to inhibit the activities of CGS, and possibly delay the growth of the mycobacterium via the disruption of methionine synthesis. Additionally, they were predicted to possess antibacterial, antimycobacterial, anti-ulcerative, and dermatological activities. The three molecules were shown to have favorable pharmacological profiles, binding energies, and binding mechanisms. Since the study is primarily computational, experimental corroboration of the results is expedient. The scaffolds of the molecules can be adapted as a skeleton for the design of next-generation CGS inhibitors for treating mycobacterial infections, especially Buruli ulcer.

Supplementary Materials: The following are available online at https:/ / www.mdpi.com/2079-3 197/9/3/32/s1, Figure S1: A heat map showing the pharmacokinetic properties of potential leads, known drugs and inhibitors predicted as GI absorption, BBB permeant, and Pgp substrate, as well as CYP1A2, CYP2C19, CYP2C9, CYP2D6, and CYP3A4 inhibitors. Red denote "Yes" whilst blue denotes "No" to cytochrome inhibition, respectively. Additionally, low Gastrointestinal (GI) absorption is denoted by green, whilst high is denoted by violet. Figure S2: Molecular mechanics Poisson-Boltzmann surface area (MM-PBSA) plot of binding free energy contribution per residue of Gentesic 5-O glucoside complex. Fluctuations by hitherto predicted critical residues are shown in red. Figure S3: Molecular mechanics Poisson-Boltzmann surface area (MM-PBSA) plot of binding free energy contribution per residue of Isoscutellarein complex. Fluctuations by hitherto predicted critical residues are shown in red. Table S1: The interaction studies of the top 24 pharmacophore hit compounds following molecular docking arranged in descending order of the number of hydrogen 
bonds formed. Known inhibitors are in red. Table S2: Physicochemical Properties of 24 ligands and 4 known anti-Buruli ulcer drugs. Some of the drugs violated Lipinski's rule. Known inhibitors, as well as known drugs, are in red. Table S3: Physicochemical properties of the top 24 ligands and 4 known anti-Buruli ulcer drugs showing other physicochemical parameters. Known inhibitors and known drugs are in red. Table S4: Toxicity results of 24 ligands with their respective structures predicted as AMES toxicity, carcinogens, and hERG I Inhibitor.

Author Contributions: S.K.K., L.M., N.N.O.D. and M.D.W. conceptualized the research project. Computational and data analysis was predominantly undertaken by S.K.K., N.N.O.D., E.D., G.M.L. with inputs from W.A.M.III, M.B.A. and M.D.W., S.K.K., N.N.O.D., E.D. and M.D.W., altogether wrote the first draft. Draft revisions and agreement on the final draft were done by all authors before submission. All authors have read and agreed to the published version of the manuscript.

Funding: There was no funding obtained for this project.

Data Availability Statement: All data and their identifications (IDs) used in this work are available in the manuscript and supplementary file.

Acknowledgments: We are grateful to the entire staff of the Biomedical Engineering Department and the Parasitology Department, University of Ghana for their invaluable support. We are also thankful to WACCBIP, University of Ghana for granting us access to the supercomputing system, Zuputo.

Conflicts of Interest: The authors declare no conflict of interest.

\section{Abbreviations}

\begin{tabular}{|c|c|}
\hline CGS & Cystathionine Gamma Synthase \\
\hline PLP & Pyridoxal Phosphate \\
\hline $\mathrm{BU}$ & Buruli Ulcer \\
\hline MetB & Methionine B \\
\hline NANPDB & North African Natural Product Database \\
\hline AfroDB & Library of natural products from African origin \\
\hline SDF & Structure Data File \\
\hline UFF & Universal Force Field \\
\hline PDB & Protein Data Bank \\
\hline $\mathrm{MD}$ & Molecular Dynamics \\
\hline $\mathrm{HPC}$ & High-Performance Computing \\
\hline GROMACS & GROningen MAchine for Chemical Simulations \\
\hline TPSA & Topological Polar Surface Area \\
\hline RMSD & Root Mean Square Deviation \\
\hline $\mathrm{EF}$ & Enrichment Factor \\
\hline ESOL & Effective Solubility \\
\hline DUD-E & Database of Useful (Docking) Decoys-Enhanced \\
\hline AMES & Salmonella typhimurium reverse mutation assay \\
\hline $\log P$ & Logarithm of the octan-1-ol/water partition coefficient \\
\hline MW & Molecular Weight \\
\hline ID & Identification \\
\hline P-gp & Permeability glycoprotein \\
\hline CYP & Cytochromes P450 \\
\hline
\end{tabular}

\section{References}

1. Portaels, F.; Silva, M.T.; Meyers, W.M. Buruli ulcer. Clin. Dermatol. 2009, 27, 291-305. [CrossRef]

2. Evans, M.R.W.; Thangaraj, H.S.; Wansbrough-Jones, M.H. Buruli ulcer. Curr. Opin. Infect. Dis. 2000, 13, 109-112. [CrossRef] [PubMed]

3. World Health Organization. Buruli ulcer disease: Mycobacterium ulcerans infection: An overview of reported cases globally. Wkly. Epidemiol. Rec. 2004, 79, 194-199.

4. Ampah, K.A.; Asare, P.; De-Graft Binnah, D.; Maccaulley, S.; Opare, W.; Röltgen, K.; Pluschke, G.; Yeboah-Manu, D. Burden and historical trend of Buruli ulcer prevalence in selected communities along the Offin River of Ghana. PLoS Negl. Trop. Dis. 2016, 10, e0004603. [CrossRef]

5. Zhang, T.; Bishai, W.R.; Grosset, J.H.; Nuermberger, E.L. Rapid assessment of antibacterial activity against Mycobacterium ulcerans by using recombinant luminescent strains. Antimicrob. Agents Chemother. 2010, 54, 2806-2813. [CrossRef] [PubMed] 
6. Merritt, R.W.; Walker, E.D.; Small, P.L.; Wallace, J.R.; Johnson, P.D.; Benbow, M.E.; Boakye, D.A. Ecology and Transmission of Buruli Ulcer Disease: A systematic review. PLoS Negl. Trop. Dis. 2010, 4, e911. [CrossRef] [PubMed]

7. Clifton, M.C.; Abendroth, J.; Edwards, T.E.; Leibly, D.J.; Gillespie, A.K.; Ferrell, M.; Dieterich, S.H.; Exley, I.; Staker, B.L.; Myler, P.J.; et al. Structure of the cystathionine $\gamma$-synthase MetB from Mycobacterium ulcerans. Acta Crystallogr. Sect. F Struct. Biol. Cryst. Commun. 2011, 67, 1154-1158. [CrossRef]

8. Berney, M.; Berney-Meyer, L.; Wong, K.W.; Chen, B.; Chen, M.; Kim, J.; Wang, J.; Harris, D.; Parkhill, J.; Chan, J.; et al. Essential roles of methionine and S-adenosylmethionine in the autarkic lifestyle of Mycobacterium tuberculosis. Proc. Natl. Acad. Sci. USA 2015, 112, 10008-10013. [CrossRef]

9. Walsh, D.S.; Portaels, F.; Meyers, W.M. Buruli Ulcer: Advances in understanding Mycobacterium ulcerans infection. Dermatol. Clin. 2011, 29, 1-8. [CrossRef]

10. Yotsu, R.R.; Richardson, M.; Ishii, N. Drugs for treating Buruli ulcer (Mycobacterium ulcerans disease). Cochrane Database Syst. Rev. 2018, 2018, CD012118. [CrossRef]

11. Zhang, Y.; MacArthur, C.; Mubila, L.; Baker, S. Control of neglected tropical diseases needs a long-term commitment. BMC Med. 2010, 8, 67. [CrossRef]

12. Kwofie, S.K.; Adobor, C.; Quansah, E.; Bentil, J.; Ampadu, M.; Miller, W.A., 3rd; Wilson, M.D. Molecular docking and dynamics simulations studies of OmpATb identifies four potential novel natural product-derived anti-Mycobacterium tuberculosis compounds. Comput. Biol. Med. 2020, 122, 103811. [CrossRef]

13. Siddiqui, A.A.; Iram, F.; Siddiqui, S.; Sahu, K. Role of natural products in drug discovery process. Int. J. Drug Dev. Res. 2014, 6, 172-204. Available online: https://www.ijddr.in/drug-development/role-of-natural-products-in-drug-discovery-process. php?aid=5524 (accessed on 15 February 2019).

14. Ekor, M. The growing use of herbal medicines: Issues relating to adverse reactions and challenges in monitoring safety. Front. Pharm. 2014, 4, 177. [CrossRef]

15. Dias, D.A.; Urban, S.; Roessner, U. A historical overview of natural products in drug discovery. Metabolites 2012, 2, 303-336. [CrossRef]

16. Berman, H.M.; Westbrook, J.; Feng, Z.; Gilliland, G.; Bhat, T.N.; Weissig, H.; Shindyalov, I.N.; Bourne, P.E. The Protein Data Bank. Nucleic Acids Res. 2000, 28, 235-242. [CrossRef] [PubMed]

17. Kleywegt, G.J.; Alwyn Jones, T. Model building and refinement practice. Methods Enzymol. 1997, 277, 208-230. [CrossRef]

18. Lill, M.A.; Danielson, M.L. Computer-aided drug design platform using PyMOL. J. Comput. Mol. Des. 2011, 25, 13-19. [CrossRef] [PubMed]

19. Yuan, S.; Chan, H.C.S.; Hu, Z. Using PyMOL as a platform for computational drug design. Wires Comput. Mol. Sci. 2017, 7, e1298. [CrossRef]

20. Bordoli, L.; Schwede, T. Automated protein structure modeling with SWISS-MODEL Workspace and the protein model portal. Methods Mol. Biol. 2012, 857, 107-136. [CrossRef] [PubMed]

21. Spoel, D.V.D.; Lindahl, E.; Hess, B.; Groenhof, G.; Mark, A.E.; Berendsen, H.J.C. GROMACS: Fast, flexible, and free. J. Comput. Chem. 2005, 26, 1701-1718. [CrossRef]

22. NCBI Resource Cordinators. Database resources of the National Center for Biotechnology Information. Nucleic Acids Res. 2013, 41, D8-D20. [CrossRef]

23. Boutet, E.; Lieberherr, D.; Tognolli, M.; Schneider, M.; Bairoch, A. UniProtKB/Swiss-Prot. In Plant Bioinformatics: Methods and Protocols; Edwards, D., Totowa, E., Eds.; Humana Press: Totowa, NJ, USA, 2007; pp. 89-112. [CrossRef]

24. Kong, Y.H.; Zhang, L.; Yang, Z.Y.; Han, C.; Hu, L.H.; Jiang, H.L.; Shen, X. Natural product juglone targets three key enzymes from Helicobacter pylori: Inhibition assay with crystal structure characterization. Acta Pharmacol. Sin. 2008, 29, 870-876. [CrossRef] [PubMed]

25. Kong, Y.; Wu, D.; Bai, H.; Han, C.; Chen, J.; Chen, L.; Hu, L.; Jiang, H.; Shen, X. Enzymatic characterization and inhibitor discovery of a new Cystathionine $\gamma$-Synthase from Helicobacter pylori. J. Biochem. 2008, 143, 59-68. [CrossRef]

26. Wolber, G.; Langer, T. LigandScout: 3-D Pharmacophores derived from protein-bound ligands and their use as virtual screening filters. J. Chem. Inf. Model. 2005, 45, 160-169. [CrossRef] [PubMed]

27. Irwin, J.J.; Shoichet, B.K. ZINC-A free database of commercially available compounds for virtual screening. J. Chem. Inf. Model. 2005, 45, 177-182. [CrossRef] [PubMed]

28. Gidaro, M.C.; Alcaro, S.; Secci, D.; Rivanera, D.; Mollica, A.; Agamennone, M.; Giampietro, L.; Carradori, S. Identification of new anti-Candida compounds by ligand-based pharmacophore virtual screening. J. Enzym. Inhib. Med. Chem. 2016, 31, 1703-1706. [CrossRef] [PubMed]

29. Lagorce, D.; Bouslama, L.; Becot, J.; Miteva, M.A.; Villoutreix, B.O. FAF-Drugs4: Free ADME-tox filtering computations for chemical biology and early stages drug discovery. Bioinformatics 2017, 33, 3658-3660. [CrossRef] [PubMed]

30. Heifets, A.; Lilien, R.H. LigAlign: Flexible ligand-based active site alignment and analysis. J. Mol. Graph. Model. 2010, $29,93-101$. [CrossRef] [PubMed]

31. Goksuluk, D.; Korkmaz, S.; Zararsiz, G.; Karaagaoglu, E.A. easyROC: An interactive web-tool for ROC curve analysis using R language environment. $R$ J. 2016, 8, 213. [CrossRef]

32. Lätti, S.; Niinivehmas, S.; Pentikäinen, O.T. Rocker: Open source, easy-to-use tool for AUC and enrichment calculations and ROC visualization. J. Cheminf. 2016, 8, 45. [CrossRef] 
33. Mysinger, M.M.; Carchia, M.; Irwin, J.J.; Shoichet, B.K. Directory of useful decoys, enhanced (DUD-E): Better ligands and decoys for better benchmarking. J. Med. Chem. 2012, 55, 6582-6594. [CrossRef]

34. Trott, O.; Olson, A.J. AutoDock Vina: Improving the speed and accuracy of docking with a new scoring function, efficient optimization and multithreading. J. Comput. Chem. 2010, 31, 455-461. [CrossRef] [PubMed]

35. Laskowski, R.A.; Swindells, M.B. LigPlot+: Multiple ligand-protein interaction diagrams for drug discovery. J. Chem. Inf. Model. 2011, 51, 2778-2786. [CrossRef] [PubMed]

36. Daina, A.; Michielin, O.; Zoete, V. SwissADME: A free web tool to evaluate pharmacokinetics, drug-likeness and medicinal chemistry friendliness of small molecules. Sci. Rep. 2017, 7, 1-13. [CrossRef]

37. Cheng, F.; Li, W.; Zhou, Y.; Shen, J.; Wu, Z.; Liu, G.; Lee, P.W.; Tang, Y. admetSAR: A comprehensive source and free tool for assessment of chemical ADMET properties. J. Chem. Inf. Model. 2012, 52, 3099-3105. [CrossRef] [PubMed]

38. Benet, L.Z.; Hosey, C.M.; Ursu, O.; Oprea, T.I. BDDCS, the Rule of 5 and drugability. Adv. Drug Deliv. Rev. 2016, 101, 89-98. [CrossRef]

39. Lagunin, A.; Stepanchikova, A.; Filimonov, D.; Poroikov, V. PASS: Prediction of activity spectra for biologically active substances. Bioinformatics 2000, 16, 747-748. [CrossRef]

40. Wishart, D.S.; Knox, C.; Guo, A.C.; Cheng, D.; Shrivastava, S.; Tzur, D.; Gautam, B.; Hassanali, M. DrugBank: A knowledgebase for drugs, drug actions and drug targets. Nucleic Acids Res. 2008, 36, D901-D906. [CrossRef]

41. Turner, P.J. XMGRACE, Version 5.1. 19. In Center for Coastal and Land-Margin Research; Oregon Graduate Institute of Science and Technology: Beaverton, OR, USA, 2005.

42. Kumari, R.; Kumar, R.; Lynn, A. g_mmpbsa-A GROMACS Tool for High-Throughput MM-PBSA Calculations. J. Chem. Inf. Model. 2014, 54, 1951-1962. [CrossRef]

43. Hevener, K.E.; Zhao, W.; Ball, D.M.; Babaoglu, K.; Qi, J.; White, S.W.; Lee, R.E. Validation of molecular docking programs for virtual screening against dihydropteroate synthase. J. Chem. Inf. Model. 2009, 49, 444-460. [CrossRef]

44. Hajian-Tilaki, K. Receiver operating characteristic (ROC) curve analysis for medical diagnostic test evaluation. Casp. J. Intern. Med. 2013, 4, 627-635. Available online: https:/ / pubmed.ncbi.nlm.nih.gov/24009950/ (accessed on 2 March 2019).

45. Greiner, M.; Pfeiffer, D.; Smith, R.D. Principles and practical application of the receiver-operating characteristic analysis for diagnostic tests. Prev. Vet. Med. 2000, 45, 23-41. [CrossRef]

46. Jiménez-Valverde, A. Insights into the area under the receiver operating characteristic curve (AUC) as a discrimination measure in species distribution modelling. Glob. Ecol. Biogeogr. 2011, 21, 498-507. [CrossRef]

47. Lasko, T.A.; Bhagwat, J.G.; Zou, K.H.; Ohno-Machado, L. The use of receiver operating characteristic curves in biomedical informatics. J. Biomed. Inform. 2005, 38, 404-415. [CrossRef] [PubMed]

48. Lewis, R.A.; Sirockin, F. 2/3D Pharmacophore definitions and their application. Ref. Modul. Chem. Mol. Sci. Chem. Eng. 2016. [CrossRef]

49. Çifci, G.; Aviyente, V.; Akten, E.D. Molecular docking study based on pharmacophore modeling for novel phosphodiesteraseiv inhibitors. Mol. Inform. 2012, 31, 459-471. [CrossRef]

50. Dallakyan, S.; Olson, A. Small-molecule library screening by docking with PyRx. Methods Mol. Biol. 2015, 1263, 243-250. [CrossRef]

51. Lionta, E.; Spyrou, G.; Vassilatis, D.K.; Cournia, Z. Structure-based virtual screening for drug discovery: Principles, applications and recent advances. Curr. Top. Med. Chem. 2014, 14, 1923-1938. [CrossRef]

52. Gimeno, A.; Ojeda-Montes, M.J.; Tomás-Hernández, S.; Cereto-Massagué, A.; Beltrán-Debón, R.; Mulero, M.; Pujadas, G.; Garcia-Vallvé, S. The Light and Dark Sides of Virtual Screening: What is there to know? Int. J. Mol. Sci. 2019, 20, 1375. [CrossRef] [PubMed]

53. Kastritis, P.L.; Bonvin, A.M.J. On the binding affinity of macromolecular interactions: Daring to ask why proteins interact. J. R. Soc. Interface 2013, 10. [CrossRef]

54. Du, X.; Li, Y.; Xia, Y.L.; Ai, S.M.; Liang, J.; Sang, P.; Ji, X.L.; Liu, S.Q. Insights into protein-ligand interactions: Mechanisms, models, and methods. Int. J. Mol. Sci. 2016, 17, 144. [CrossRef]

55. Herschlag, D.; Pinney, M.M. Hydrogen bonds: Simple after all? Biochemistry 2018, 57, 3338-3352. [CrossRef] [PubMed]

56. Bulusu, G.; Desiraju, G. Strong and weak hydrogen bonds in protein-ligand recognition. J. Indian Inst. Sci. 2019, $100,31-41$. [CrossRef]

57. Hubbard, R.; Haider, M. Hydrogen bonds in proteins: Role and strength. Encycl. Life Sci. 2010, 1. [CrossRef]

58. Schreiber, G.; Keating, A.E. Protein binding specificity versus promiscuity. Curr. Opin. Struct. Biol. 2011, 21, 50-61. [CrossRef] [PubMed]

59. Wang, G.Y.; Zheng, H.H.; Zhang, K.Y.; Yang, F.; Kong, T.; Zhou, B.; Jiang, S.X. The roles of cytochrome P450 and P-glycoprotein in the pharmacokinetics of florfenicol in chickens. Iran. J. Vet. Res. 2018, 19, 9-14. Available online: https://www.ncbi.nlm.nih.gov/ pmc/articles/PMC5960766/ (accessed on 19 June 2019).

60. Devadasu, V.R.; Deb, P.K.; Maheshwari, R.; Sharma, P.; Tekade, R.K. Physicochemical, pharmaceutical, and biological considerations in GIT absorption of drugs. In Dosage Form Design Considerations; Tekade, R.K., Ed.; Academic Press: Cambridge, MA, USA, 2018; pp. 149-178.

61. Suenderhauf, C.; Hammann, F.; Huwyler, J. Computational prediction of blood-brain barrier permeability using decision tree induction. Molecules 2012, 17, 10429-10445. [CrossRef] 
62. Samiei, M.; Asgary, S.; Farajzadeh, M.; Bargahi, N.; Abdolrahimi, M.; Kananizadeh, U.; Dastmalchi, S. Investigating the mutagenic effects of three commonly used pulpotomy agents using the ames test. Adv. Pharm. Bull. 2015, 5, 121-125. [CrossRef]

63. Wang, W.Q.; Duan, H.X.; Pei, Z.T.; Xu, R.R.; Qin, Z.T.; Zhu, G.C.; Sun, L.W. Evaluation by the Ames assay of the mutagenicity of UV filters using benzophenone and benzophenone-1. Int. J. Environ. Res. Public Health 2018, 15, 1907. [CrossRef]

64. Yasuda, C.; Yasuda, S.; Yamashita, H.; Okada, J.; Hisada, T.; Sugiura, S. The human ether-a-go-go-related gene (hERG) current inhibition selectively prolongs action potential of midmyocardial cells to augment transmural dispersion. J. Physiol. Pharm. 2015, 66, 599-607. Available online: https:/ / pubmed.ncbi.nlm.nih.gov/26348084/ (accessed on 21 June 2019).

65. Danker, T.; Möller, C. Early identification of hERG liability in drug discovery programs by automated patch clamp. Front. Pharm. 2014, 5, 203. [CrossRef] [PubMed]

66. Guha, R. On exploring structure activity relationships. Methods Mol. Biol. 2013, 993, 81-94. [CrossRef]

67. Benchabane, Y.; Di Giorgio, C.; Boyer, G.; Sabatier, A.S.; Allegro, D.; Peyrot, V.; De Méo, M. Photo-inducible cytotoxic and clastogenic activities of 3,6-di-substituted acridines obtained by acylation of proflavine. Eur. J. Med. Chem. 2009, 44, 2459-2467. [CrossRef] [PubMed]

68. Stepanchikova, A.V.; Lagunin, A.A.; Filimonov, D.A.; Poroikov, V.V. Prediction of Biological Activity Spectra for Substances: Evaluation on the Diverse Sets of Drug-Like Structures. 2003. Available online: https://www.ingentaconnect.com/content/ben/ $\mathrm{cmc} / 2003 / 00000010 / 00000003 /$ art00003 (accessed on 8 May 2019).

69. Tsouh Fokou, P.V.; Nyarko, A.K.; Appiah-Opong, R.; Tchokouaha Yamthe, L.R.; Ofosuhene, M.; Boyom, F.F. Update on medicinal plants with potency on Mycobacterium ulcerans. Biomed. Res. Int. 2015, 2015, 1-16. [CrossRef]

70. Kwofie, S.K.; Dankwa, B.; Odame, E.A.; Agamah, F.E.; Doe, L.; Teye, J.; Agyapong, O.; Miller, W.A., 3rd; Mosi, L.; Wilson, M.D. In Silico screening of isocitrate lyase for novel anti-buruli ulcer natural products originating from Africa. Molecules 2018, 23, 1550. [CrossRef]

71. Dunn, M.F.; Ramírez-Trujillo, J.A.; Hernández-Lucas, I. Major roles of isocitrate lyase and malate synthase in bacterial and fungal pathogenesis. Microbiology 2009, 155, 3166-3175. [CrossRef] [PubMed]

72. Lirio, S.B.; Macabeo, A.P.; Paragas, E.M.; Knorn, M.; Kohls, P.; Franzblau, S.G.; Wang, Y.; Aguinaldo, M.A. Antitubercular constituents from Premna odorata Blanco. J. Ethnopharmacol. 2014, 154, 471-474. [CrossRef]

73. Sasikumar, K.; Ghosh, A.R.; Dusthackeer, A. Antimycobacterial potentials of quercetin and rutin against Mycobacterium tuberculosis H37Rv 3. Biotech 2018, 8, 427. [CrossRef]

74. Butova, T.; Zaitseva, S.; Butov, D.; Stepanenko, G. Morphological changes in experimental tuberculosis resulting from treatment with quercetin and polyvinylpyrrolidone. Int. J. Mycobacteriol. 2016, 5, S103-S104. [CrossRef] [PubMed]

75. Hughes, W.T.; Dankner, W.M.; Yogev, R.; Huang, S.; Paul, M.E.; Flores, M.A.; Kline, M.W.; Wei, L.J. Pediatric AIDS Clinical Trials Group 254 Team. Comparison of atovaquone and azithromycin with trimethoprim-sulfamethoxazole for the prevention of serious bacterial infections in children with hiv infection. Clin. Infect. Dis. 2005, 40, 136-145. [CrossRef] [PubMed]

76. Molecular Docking, Estimating Free Energies of Binding, and AutoDock's Semi-Empirical Force Field. Dr. Sebastian Raschka. 2014. Available online: https://sebastianraschka.com/Articles/2014_autodock_energycomps.html (accessed on 10 February 2020).

77. Genheden, S.; Ryde, U. The MM/PBSA and MM/GBSA methods to estimate ligand-binding affinities. Expert Opin. Drug Discov. 2015, 10, 449-461. [CrossRef] [PubMed]

78. Kwofie, S.K.; Broni, E.; Teye, J.; Quansah, E.; Issah, I.; Wilson, M.D.; Miller, W.A., 3rd; Tiburu, E.K.; Bonney, J. Pharmacoinformaticsbased identification of potential bioactive compounds against Ebola virus protein VP24. Comput. Biol. Med. 2019, 113, 103414. [CrossRef] [PubMed] 